\title{
Holistic Resource Management for Sustainable and Reliable Cloud Computing: An Innovative Solution to Global Challenge
}

\author{
Sukhpal Singh Gill ${ }^{1,2}$, Peter Garraghan ${ }^{1}$, Vlado Stankovski ${ }^{3}$, Giuliano Casale ${ }^{4}$, Ruppa K. Thulasiram ${ }^{5}$, Soumya K. \\ Ghosh $^{6}$, Kotagiri Ramamohanarao ${ }^{2}$ and Rajkumar Buyya ${ }^{2}$ \\ ${ }^{1}$ School of Computing and Communications, Lancaster University, UK \\ ${ }^{2}$ Cloud Computing and Distributed Systems (CLOUDS) Laboratory, \\ School of Computing and Information Systems, The University of Melbourne, Australia \\ ${ }^{3}$ Faculty of Civil and Geodetic Engineering, University of Ljubljana, Ljubljana, Slovenia \\ ${ }^{4}$ Department of Computing, Imperial College London, London, UK \\ ${ }^{5}$ Department of Computer Science, University of Manitoba, Canada \\ ${ }^{6}$ Department of Computer Science and Engineering, Indian Institute of Technology, Kharagpur, India \\ s.s.gill1@lancaster.ac.uk, p.garraghan@lancaster.ac.uk, vlado.stankovski@fgg.uni-lj.si, g.casale@imperial.ac.uk, tulsi@cs.umanitoba.ca, \\ skg@cse.iitkgp.ac.in, kotagiri@unimelb.edu.au, rbuyya@ unimelb.edu.au
}

\begin{abstract}
Minimizing the energy consumption of servers within cloud computing systems is of upmost importance to cloud providers towards reducing operational costs and enhancing service sustainability by consolidating services onto fewer active servers. Moreover, providers must also provision high levels of availability and reliability, hence cloud services are frequently replicated across servers that subsequently increases server energy consumption and resource overhead. These two objectives can present a potential conflict within cloud resource management decision making that must balance between service consolidation and replication to minimize energy consumption whilst maximizing server availability and reliability, respectively. In this paper, we propose a cuckoo optimization-based energy-reliability aware resource scheduling technique (CRUZE) for holistic management of cloud computing resources including servers, networks, storage, and cooling systems. CRUZE clusters and executes heterogeneous workloads on provisioned cloud resources and enhances the energy-efficiency and reduces the carbon footprint in datacenters without adversely affecting cloud service reliability. We evaluate the effectiveness of CRUZE against existing stateof-the-art solutions using the CloudSim toolkit. Results indicate that our proposed technique is capable of reducing energy consumption by $20.1 \%$ whilst improving reliability and CPU utilization by $17.1 \%$ and $15.7 \%$ respectively without affecting other Quality of Service parameters.
\end{abstract}

Keywords: Cloud Computing, Energy Consumption, Sustainability, Reliability, Holistic Management, Cloud Datacenters

\section{Introduction}

Commercial cloud providers such as Microsoft, Google, and Amazon heavily depend on datacenters to support the ever-increasing demand for computational requirements of their services. Such demand has subsequently increased operational costs of running large infrastructures, as well as producing substantial carbon emissions that negatively impact the environmental sustainability of cloud services [1]. Existing efforts to tackle this problem primarily focus on minimizing the energy consumption of servers via service consolidation to reduce the number of active servers and increase datacenter resource utilization [1] [2] [3]. However, such approaches typically do not consider other core datacenter components, including the network, storage, and cooling systems, which constitute significant amount (32\% approximately) of total Cloud DataCentres (CDC) power consumption [41]. Server consolidation unaware of the cooling system may increase the number of hot spots in the datacenter which subsequently increases the requirement of cooling capacity and reduces cooling efficiency [43]. Hotspot mitigation is performed via load balancing. However, load balancing can widely spread communicating Virtual Machines (VMs) across multiple hosts without considering their pairwise network traffic, increasing network cost and energy consumption [50]. Thus, to create cloud platforms that are energy efficient, a resource management approach capable of managing all these resources (network, storage, servers, memory and cooling components) in a holistic manner is required [2].

While energy efficiency is critical, cloud providers must also provide highly available and reliable cloud services [2]. However, with the growing adoption of cloud, CDCs are rapidly expanding in terms of scale and system complexity, which has subsequently increased the frequency and diversity of failures [42]. These failures range across Service Level Agreement (SLA) violation, data corruption, loss/premature execution termination, degrading cloud service 
performance and availability [3]. In order to address this problem, one of the most common practices is to replicate cloud services frequently to minimize the risk of simultaneous failure [46]. However, replicas require additional resource usage from servers within the CDC leading to extra resource usage which increases their respective power consumption [42]. It has been reported that a huge quantity of base-load energy is consumed even when actual cloud resource usage is very low or even idle [4] [5]. This results in a potential conflict in resource management decision making within the CDC to balance between energy consumption and reliability [57] [58]. In order to minimize server power usage, it would be preferable to consolidate servers and power down idle machines [59]. On the other hand, in order to maximize cloud service availability and reliability, replicas across additional machines are required [44]. Therefore, a holistic resource management is not only required for managing all aforementioned components, but must also consider replication and coordination of services to enable reliable delivery of cloud services in a costefficient manner.

Heuristic methods such as evolutionary algorithm are a suitable candidate to tackle the complexity of this problem. Li et al. [6] suggested that scheduling algorithms equipped with a Cuckoo Optimization (CO) algorithm can be used in this regard because CO algorithm performs better than Particle Swarm Optimization (PSO) and Ant Colony Optimization (ACO) in terms of accuracy, speed and convergence [29] for solving batch process and job scheduling problems [28] [29]. The main motivation of this research work is to extend a conceptual model proposed in [2] [44] to develop a Cuckoo optimization based efficient Resource UtiliZation techniquE called CRUZE for holistic management of all resources (servers, network, storage, cooling systems) to improve the energy efficiency and reduce carbon footprint while minimizing service failure within hardware, service, and software to maintain required cloud service reliability. Our approach clusters the workloads based on their Quality of Service (QoS) parameters for provisioning of cloud resources and schedules the provisioned resources for workload execution using a Cuckoo optimization-based scheduling algorithm.

The rest of the article is structured as follows. Section 2 presents the related work of existing holistic resource management approaches. Section 3 presents the system model and design model of CRUZE. Section 4 proposes the resource provisioning and scheduling strategy. Section 5 describes the experimental setup and presents the results and analysis. Section 6 summarizes the paper and offers future research directions.

\section{Related Work}

Holistic management of cloud resources is a challenging task due to dynamic requirements of cloud users [53]. The majority of existing works study the energy management of servers alone without omitting other components, which consume substantial energy [9] [55]. This section describes the existing resource management techniques and their comparison with our proposed approach.

\subsection{Energy-aware Cloud Resource Scheduling}

Li et al. [6] proposed an Energy-Aware Resource Scheduling (EARS) technique to execute workloads within virtual cloud environments. EARS technique models the power and failure profiles for CDC and implements them using event-based cloud simulator, and is an effective in reducing energy cost of cloud data center and improving task completion rate, however only considers homogeneous workloads. Similarly, Li et al. [8] proposed a VM Scheduling (VMS) technique to reduce energy consumption of servers and identifies the effect of energy consumption on SLA violation rate to improve user satisfaction. Balis et al. [7] proposed a Holistic Approach (HA) for management of IT infrastructure to reduce execution cost and energy consumption. Perez et al. [10] proposed a Holistic Workload Scaling (HWS) technique to enable scaling of resources vertically as well as horizontally simultaneously and aids to reduce latency using multi-scaling approach without considering energy consumption of cloud resources. Luo et al. [11] formulates energy consumption as a task-core assignment and scheduling problem and proposed a Holistic Energy Optimization Framework (HEOF) to reduce thermal effect as well as cooling cost simultaneously and HEOF framework focuses on powerful computation capability. Liu et al. [12] proposed a Server-based Cloud-enabled Architecture (SCA) to improve the energy-efficiency of different hardware components such as memory, storage and processors. Furthermore, the performance of SCA is evaluated using a case study of video tracking application and experimental results show that SCA performs better in terms of memory utilization. Guzek et al. [13] proposed a Holistic Model for Resource Management (HMRM) in virtualization based cloud datacenter to reduce the energy consumption of different resources such as memory, storage and networking. Ferrer et al. [14] proposed a Holistic Approach for Cloud Service Provisioning (HACSP) to meet predicted and unforeseen changes in resource requirements dynamically and optimizes energy cost. Feller et al. [15] proposed a Holistic approach for Energy 
Management (HEM) technique for effective management of virtual cloud resources using dynamic web workloads and saves the substantial amount of energy. Dinkar et al. [60] proposed Energy Efficient Data Center Management (EEDCM) technique under availability constraints and outlines the importance of availability and designs a hill climbing algorithm to prevent failure zone. The experimental result shows that EEDCM technique reduces the energy consumption by the datacenter, but the trade-off between energy consumption and other important QoS parameters such as reliability, cost and execution time are omitted.

\subsection{Reliability-aware Cloud Resource Scheduling}

Zhou et al. [16] presented a Cloud Service Reliability Enhancement (CSRE) approach to utilization of network and storage resources. This approach stores the state of VM using service checkpointing, which is in executing state. Further, node failure predicator is designed to optimize the use of network resources. Li et al. [17] developed a convergent dispersal based multi-cloud storage (CDStore) solution to offer reliable, secure and cost-efficient cloud service. The proposed solution offers deterministic-based deduplication approach to save network bandwidth and storage space. Moreover, CDStore uses two-stage deduplication to protect the system from malicious attacks. Azimzadeh et al. [18] proposed a Multi-Objective Resource Scheduling (MORS) approach to increase the reliability of cloud service and optimize the execution time. Further, authors identify a trade-off between reliability and execution time for efficient execution of HPC (High Performance Computing) workloads. Poola et al. [19] proposed an Adaptive and Just-In-Time (AJIT) scheduling approach using spot and on-demand instances to provide fault management mechanism. This approach uses resource consolidation to optimize execution time and cost and experimental results indicates that AJIT is efficient for execution of deadline-oriented workloads. Qu et al. [20] proposed a Heterogeneous Spot Instances-based Auto-scaling (HSIA) approach to execute web applications in a reliable manager. Further, HSIA approach designed a fault tolerant system to improve the availability and reduce the execution cost and response time of cloud service. Liu et al. [21] proposed a replication-based state management (E-Storm) approach actively maintains multiple state backups on different VMs during the execution of real-world and synthetic streaming applications. The performance of E-Storm is evaluated against checkpointing method and experimental results indicates that E-Storm achieves effective results in terms of latency and throughput. Abdulhamid et al. [22] proposed a Dynamic Clustering League Championship Approach (DCLCA) to minimize fault reduction in task failure during resource scheduling for workload execution. Liang et al. [61] proposed a Resource Optimization method for Cloud Data Center (ROCDC), which designs a conceptual model to optimize the performance parameters reliability and energy while scheduling resources. However, this approach was not validated via simulation or experimentation.

\subsection{Cuckoo Optimization based Energy-aware Cloud Resource Scheduling}

Shahdi-Pashaki et al. [23] proposed a Group Technology-based model and Cuckoo Optimization (GTCO) algorithm to allocate resources for effective mapping of VMs to cloud workloads. GTCO reduces energy cost during execution of VMs and performs better than round robin based resource scheduling. Sundarrajan and Vasudevan [24] proposed a Cuckoo Optimization based Task Scheduling (COTC) algorithm to schedule the tasks in cloud processing and improves the energy utilization for the execution of homogeneous workloads. Abbasi and Mohri [25] proposed a Cuckoo Optimization based Resource Management (CORM) mechanism for task scheduling, which improves load balancing to reduce energy cost. CORM improves energy-efficiency during execution of cloud resources and performs better than round robin based resource scheduling. Navimipour and Milani [26] proposed a Cuckoo Search Algorithm based Task Scheduling (CSATS) technique for effective utilization of cloud resources. Authors just measured the fitness value (execution time) of CSATS with different values of probability to find the cloud resource for execution of workloads. Madni et al. [27] proposed a Cuckoo Search Meta-Heuristic (CSMH) algorithm, which optimizes energy consumption of cloud workloads. The performance of COTC [24] and CSMH [27] have been evaluated using CloudSim [30] and both reduces energy cost of servers without focusing on other components of the cloud datacenter.

\subsection{Comparison of CRUZE with Existing Resource Scheduling Techniques}

Table 1 compares our proposed technique (CRUZE) with existing resource scheduling approaches discussed above. We identified that existing approaches for holistic resource management only consider one or two components simultaneously. The majority of existing work schedule resources for the execution of homogeneous workloads while others like EARS [6], HEOF [11] and AJIT [19] schedule resources for the execution of heterogeneous workloads as well. None of the existing works considers clustering of workloads for resource provisioning. Provisioning based resource scheduling is only considered in CSRE [16]. Cuckoo Optimization (CO) based scheduling is performed in 
GTCO [23], COTC [24], CORM [25], CSATS [26] and CSMH [27], but scheduled resources only for homogenous cloud workload without any provisioning of resources. GTCO [23] optimizes energy cost and latency but COTC [24], CORM [25], CSATS [26] and CSMH [27] only optimizes energy cost and execution time. This is the first research paper which focuses on holistic management of core CDC components towards providing reliable and sustainable cloud services. The proposed technique (CRUZE) schedules the provisioned resources for the execution of clustered and heterogeneous workloads to enable reliable and sustainable cloud services.

Table 1: Comparison of CRUZE with existing holistic resource management techniques

\begin{tabular}{|c|c|c|c|c|c|c|c|c|c|c|c|c|c|}
\hline \multirow{3}{*}{ Technique } & \multicolumn{13}{|c|}{ Components of Holistic Resource Management for Cloud Computing } \\
\hline & \multirow{2}{*}{1} & \multirow{2}{*}{2} & \multirow{2}{*}{$\mathbf{3}$} & \multirow{2}{*}{4} & \multicolumn{9}{|c|}{ Sustainability-aware and Reliability-aware Holistic Management } \\
\hline & & & & & 5 & 6 & 7 & 8 & 9 & 10 & 11 & 12 & 13 \\
\hline EARS [6] & & & & & & $\checkmark$ & & $\checkmark$ & & $\checkmark$ & & & \\
\hline HA [7] & & & & & & $\checkmark$ & & $\checkmark$ & & & & & \\
\hline VMS [8] & & & & & & & & $\checkmark$ & & & $\checkmark$ & & \\
\hline HWS [10] & & & & & & $\sqrt{ }$ & & & & & & & \\
\hline HEOF [11] & $\sqrt{ }$ & & & & & & & $\checkmark$ & $\checkmark$ & $\checkmark$ & & $\checkmark$ & $\checkmark$ \\
\hline SCA [12] & & & & & & & & $\checkmark$ & & & & & \\
\hline HMRM [13] & & & & & & & & $\checkmark$ & & & $\checkmark$ & & \\
\hline HACSP [14] & & & & & & & & $\checkmark$ & & & & & \\
\hline HEM [15] & & & & & & & & $\checkmark$ & & & $\checkmark$ & & \\
\hline CSRE [16] & & & $\checkmark$ & & $\checkmark$ & & & & & & $\checkmark$ & & \\
\hline CDStore [17] & & & & & $\checkmark$ & $\checkmark$ & & & & & & & \\
\hline MORS [18] & & & & & $\sqrt{ }$ & $\checkmark$ & & & & & & & \\
\hline AJIT [19] & $\checkmark$ & & & & $\checkmark$ & $\checkmark$ & & & & & & & \\
\hline HSIA [20] & & & & & $\checkmark$ & $\checkmark$ & & & & & & & \\
\hline E-storm [21] & & & & & $\checkmark$ & $\checkmark$ & & & & & & & \\
\hline DCLCA [22] & & & & & $\checkmark$ & $\checkmark$ & & & & & & & \\
\hline GTCO [23] & & & & $\checkmark$ & & & & $\checkmark$ & & & $\checkmark$ & & \\
\hline COTC [24] & & & & $\sqrt{ }$ & & & & $\checkmark$ & & & & & \\
\hline CORM [25] & & & & $\checkmark$ & & $\checkmark$ & & $\checkmark$ & & & & & \\
\hline CSATS [26] & & & & $\checkmark$ & & $\checkmark$ & & & & & & & \\
\hline CSMH [27] & & & & $\checkmark$ & & $\checkmark$ & & & & & & & \\
\hline EEDCM [60] & & & & & & & & $\checkmark$ & & & & & \\
\hline ROCDC [61] & & & & & $\checkmark$ & & & $\checkmark$ & & & & & \\
\hline CRUZE (proposed) & $\checkmark$ & $\checkmark$ & $\checkmark$ & $\boldsymbol{V}$ & $\boldsymbol{J}$ & $\checkmark$ & $\checkmark$ & $\boldsymbol{J}$ & $\checkmark$ & $\sqrt{ }$ & $\boldsymbol{J}$ & $\checkmark$ & $\mathbf{I}$ \\
\hline
\end{tabular}

Abbreviations: 1 - Heterogeneous Workloads, 2 - Workload Clustering, 3 - Provisioning based Scheduling, 4 - Cuckoo Optimization Scheduling, 5 - Failures, 6 - Application QoS, 7 - Capacity Planning, 8 - Energy Management, 9 - Thermal-aware Scheduling, 10 - Cooling, 11 - Virtualization, 12 - Renewable Energy and 13 - Waste Heat Utilization.

\section{System Model}

As our proposed approach operates within a holistic CDC [2], we present and describe all core components within the system as shown in Figure 1.Our approach considers components within all levels of cloud service provisioning.

1. Software as a Service (SaaS): This layer handles the incoming user workloads (batch style or interactive) and forward those workloads (requests or user sessions) to workload manager as discussed in Section 4.1. Based on their QoS requirements such as deadlines and budget constraints, workload manager maintains the queue of workloads in a specific order based on their priorities.

2. Platform as a Service (PaaS): This layer deploys a controller to handle the different functions of the system. Controller schedules the provisioned cloud resources efficiently with three main objectives: 1) maximize resource utilization, 2) minimize energy consumption and 3) improve the reliability and sustainability of cloud datacenters. Further, a controller (middleware) has five sub modules: cooling manager, energy manager, fault manager, $\mathrm{VM} /$ resource manager and workload manager and roles of sub modules is described below: 
a) Workload manager maintains the queue of arriving user workloads from the application manager and recognizes their QoS constraints and forwards the QoS information to the next module i.e. VM/resource manager for provisioning and scheduling of resources.

b) $V M$ /resource manager schedules the provisioned resources for their execution using virtual or physical machines based on QoS requirements of workload.

c) Fault manager performs fault detection and correction with the minimal performance degradation. We have considered three types of faults for this research work: VM creation failures, host failures (Processing Elements failure and memory failure) and high-level failures like cloudlets failures (which are caused by any networking problem) [44]. FIM-SIM [45] is integrated with CloudSim toolkit [30] to simulate failures as discussed in Section 5.

d) Data Center (DC) Manager acts as a broker to handle other modules such as cooling manager and energy manager for cooling and energy management respectively.

The working of controller (CRUZE) is discussed in Section 4 in detail.

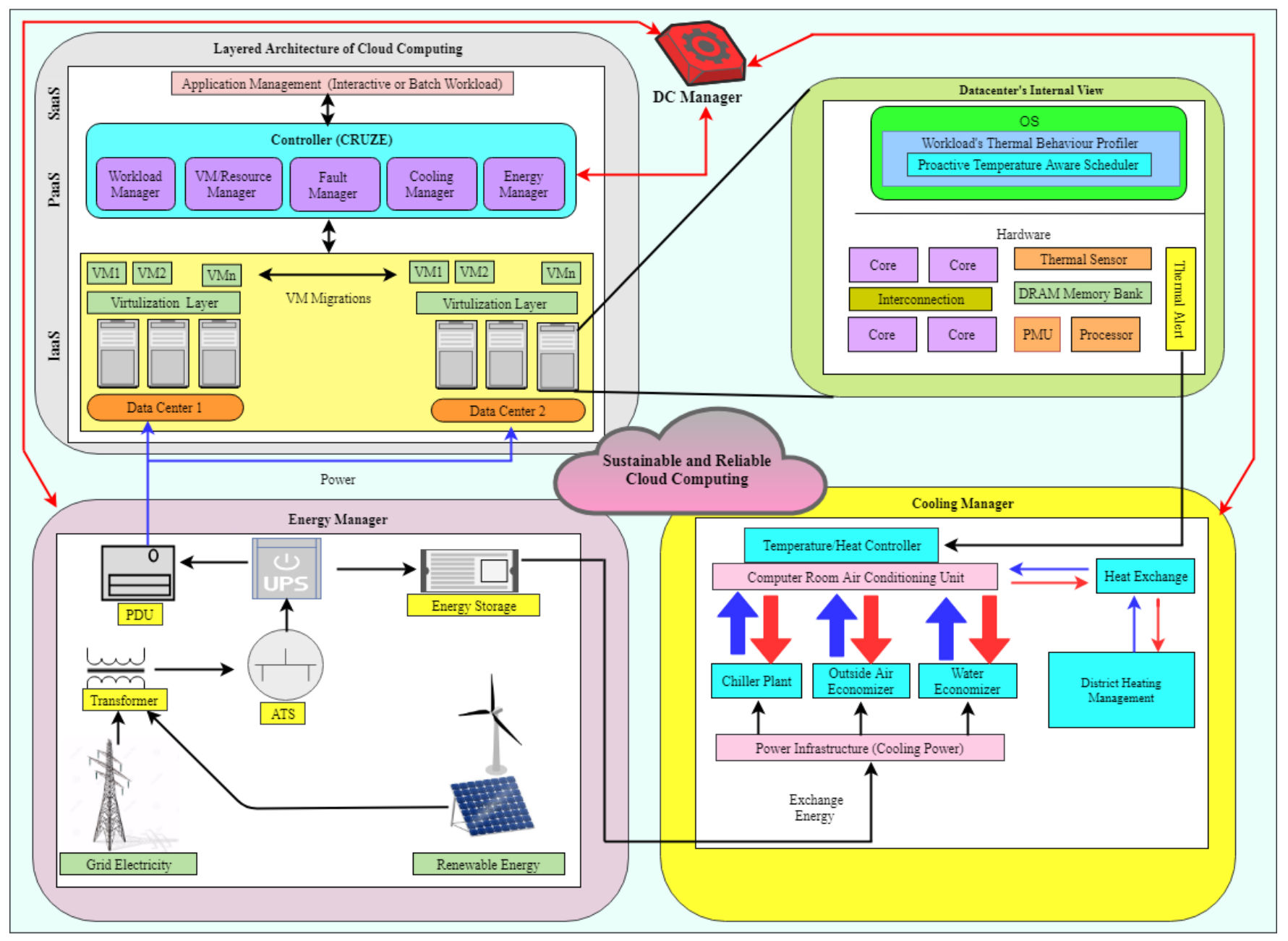

Figure 1: System Model

3. Infrastructure as a Service (IaaS): IaaS layer comprises of information related to cloud infrastructure such as VMs and CDCs. Furthermore, the virtualization layer enables workload balancing via VM migration. The variations of the temperature of different VMs running at different cores is measured, monitored and controlled by proactive temperature-aware scheduler. Power Management Unit (PMU) is deployed to provide and control the power for different components of cloud data centers. Check-pointing mechanism is provided by Dynamic Random-Access Memory (DRAM) by storing the current states of VMs [46]. Thermal sensors monitor the value 
of temperature and forward to the Thermal profiling and monitoring module to analyze the temperature variations of cloud datacenters. When system generates thermal alert then heat controller takes a required action to control the temperature if it is higher than its threshold value and maintains the performance of datacenter. Uninterruptible Power Supply (UPS) is deployed to continue the power in case of power failure from main sources. For cooling management, the district heating management uses water economizer, outside air economizer and chiller plant to control the temperature of CDC. Energy manager manages the energy produced from renewable and nonrenewable sources. Sustainable CDCs focuses more on renewable energy sources (solar and wind) [2] [52]. To provide reliable services, CDC can prefer grid energy for the execution of deadline-aware workloads. Automatic Transfer Switch (ATS) manages the electricity producing from renewable as well as non-renewable sources. Moreover, Power Distribution Unit (PDU) transfers the energy to all the devices of cloud datacenters and cooling components.

\subsection{Design Models}

We have used following design models for holistic management of cloud resources:

a) Energy Model: The energy model is developed on the basis that resource utilization has a linear relationship with energy consumption [6] [7] [39] [46] [67] [68]. Energy Consumption (E) of a CDC can be expressed as [Eq. 1]:

$$
E=E_{\text {Processor }}+E_{\text {Storage }}+E_{\text {Memory }}+E_{\text {Network }}+E_{\text {Cooling }}+E_{\text {Extra }}
$$

$E_{\text {Processor }}$ represents the processor's energy consumption, which is calculated using [Eq. 2]:

$$
E_{\text {Processor }}=\sum_{r=1}^{r=\text { cores }}\left(E_{\text {dynamic }}+E_{S C}+E_{\text {Leakage }}+E_{\text {idle }}\right)
$$

where $E_{\text {dynamic }}$ represents dynamic energy consumption and calculated using [Eq. 3], $E_{S C}$ represents shortcircuit energy consumption, $E_{\text {Leakage }}$ represents power loss due to transistor leakage current and $E_{i d l e}$ represents the energy consumption when processor component is idle.

$$
E_{\text {dynamic }}=C V^{2} f
$$

where $C$ is capacitance, $f$ is frequency, and $V$ is voltage.

$E_{\text {Storage }}$ represents the energy consumption of storage device, which performs data read and write operations and it is calculated using [Eq. 4]:

$$
E_{\text {Storage }}=E_{\text {Readoperation }}+E_{\text {Writeoperation }}+E_{\text {idle }}
$$

where $E_{\text {idle }}$ represents the energy consumption when storage component is idle.

$E_{\text {Memory }}$ represents the energy consumption of the main memory (RAM/DRAM) and cache memory (SRAM), which is calculated using [Eq. 5]:

$$
E_{\text {Memory }}=E_{S R A M}+E_{D R A M}
$$

$E_{\text {Network }}$ represents the energy consumption of networking equipment such as routers, switches and gateways, LAN cards, which is calculated using [Eq. 6]:

$$
E_{\text {Network }}=E_{\text {Router }}+E_{\text {Gateway }}+E_{\text {LANcard }}+E_{\text {Switch }}
$$

$E_{\text {Cooling }}$ represents the energy is consumed by cooling devices (air conditioners, compressors and fans) to maintain the temperature of cloud datacenter [6], which is calculated using [Eq. 7]:

$$
E_{\text {Cooling }}=E_{A C}+E_{\text {Compressor }}+E_{\text {Fan }}
$$

$E_{\text {Extra }}$ represents the energy consumption of other parts, including the current conversion loss and others, which is calculated using [Eq. 8]:

$$
E_{\text {Extra }}=E_{\text {Motherboard }}+\sum_{f=0}^{F} E_{\text {connector }, f}
$$


where $E_{\text {Motherboard }}$ is energy consumed by motherboard (s) and $\sum_{f=0}^{F} E_{\text {connector, } f}$ is energy consumed by a connector (port) running at the frequency $f$.

For a resource $r_{k}$ at given time $t$, the resource utilization $R E S U_{t, k}$ is defined as [Eq. 9]:

$$
R E S U_{t, k}=\sum_{i=1}^{m} r u_{t, k, i}
$$

where $m$ is the number of cloud workloads running at time $t, r u_{t, k, i}$ is the resource (VMs) usage of workload $w_{i}$ on resource $r_{k}$ at given time $t$. The actual energy consumption $\left(E_{\text {consumption }}\right)$ is $E C O N_{t, k}$ of a resource $r_{k}$ at given time $t$ is defined as [Eq. 10]:

$$
E_{\text {consumption }}=E \operatorname{CON}_{t, k}=\left(E_{\max }-E_{\min }\right) \times R E S U_{t, k}+E_{\min }
$$

where $E_{\max }$ is the energy consumption at the peak load (or $100 \%$ utilization) and $E_{\min }$ is the minimum consumption of energy in the idle/active mode (or as low as $1 \%$ utilization), which can be calculated using [Eq. 1] through profiling.

b) Reliability Model: Reliability of cloud services is the ability to provision correct service [44] [46], and is calculated as [Eq. 11]:

$$
R_{\text {service }}=e^{-\lambda t}
$$

where $t$ is time for the resource to deal with its request for any workload execution and $\lambda$ is the failure rate of the resource at the given time, which is calculated using [Eq. 13].

The list of available SLAs $=\left\langle m_{1}, m_{2} \ldots \ldots \ldots \ldots \ldots m_{n}\right\rangle$, where $n$ is the total number of SLAs.

$$
\text { Failure }(m)= \begin{cases}1, & m \text { is not violated } \\ 0, & m \text { is violated }\end{cases}
$$

Failure rate $(\lambda)$ is computed as a ratio of the summation of all the SLA violated to the total number of SLAs [46].

$$
\lambda=\sum_{i=1}^{n}\left(\frac{\text { Failure }\left(m_{i}\right)}{n}\right)
$$

c) Capacity Planning Model: The capacity model is defined in terms of memory utilization, disk utilization and network utilization at given time $t$ [33]. The formula for calculating memory utilization $\left(M_{U t i l i z a t i o n}\right)$ in percentage is as follows [Eq. 14]:

$$
M_{\text {Utilization }}=\frac{\text { Total Physical Memory }-(\text { Memory Free }+ \text { Memory Buffers }+ \text { Cache Memory })}{\text { Total Physical Memory }} \times 100
$$

The formula for calculating disk utilization $\left(D_{\text {Utilization }}\right)$ in percentage is as follows [Eq. 16]:

$$
\begin{gathered}
D_{\text {Usage }}=\frac{\text { Total Used }}{\text { Total } \mathrm{HD} \text { size }} \times 100 \\
D_{\text {Utilization }}=\frac{\text { Storage Allocation Units } \times \text { Storage Used }}{\text { StorageAllocationUnits } \times \text { Storage Size }} \times 100
\end{gathered}
$$

The formula for calculating network utilization $\left(N_{\text {Utilization }}\right)$ in percentage is as follows [Eq. 17]:

$$
N_{\text {Utilization }}=\frac{\text { data bits }}{\text { bandwidth } \times \text { interval }} \times 100
$$

d) Temperature Model: We used Computer Room Air Conditioning (CRAC) model and RC (where R and C are thermal resistance $(\mathrm{k} / \mathrm{w})$ and heat capacity $(\mathrm{j} / \mathrm{k})$ of the host respectively) thermal model [34] [64] [65] [66]

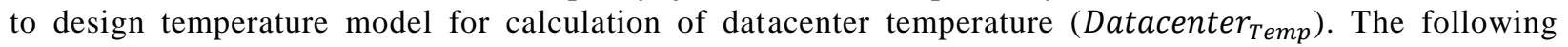
formula is used to calculate the temperature of datacenter [Eq. 18]. 


$$
\text { Datacenter }_{\text {Temp }}=\text { Temp }_{\text {inlet }}+\sum_{i=1}^{n}\left(\frac{\text { Temp }_{C P U_{i}}}{n}\right)+T_{\text {initial } \times} e^{-\mathrm{RC}}
$$

where CRAC model is used to calculate inlet temperature ( Temp $\left._{\text {inlet }}\right)$ and RC model is used to calculate CPU temperature $\left(T e m p_{C P U}\right) . T_{\text {initial }}$ is the initial temperature of the CPU. $1 \leq \mathrm{i} \leq n, n$ is the number of CPUs.

e) Renewable Energy Model: A renewable energy model [35] is used in terms of Energy Reuse Effectiveness (ERE) and [Eq. 19] is used to calculate its value.

$$
\mathrm{ERE}=\frac{E-\text { Energy }_{\text {Reused }}}{E}
$$

The value of $E$ is calculated using [Eq. 1]. Energy $y_{\text {Reused }}$ is amount of energy reused by different IT equipment.

f) Waste Heat Utilization Model: The district heating management based waste heat utilization model [36] is used in terms of recirculation ratio $\left(R_{R}\right)$ and it is defined as the following [Eq. 20]:

$$
R_{R}=\frac{W_{m}}{W_{s}}
$$

where $W_{m}=$ mass flow rate of the water entering the circulation system, kilograms per second $(\mathrm{kg} / \mathrm{s})$ and $W_{s}$ $=$ mass flow rate of the steam generated in the circulation system, $\mathrm{kg} / \mathrm{s}$. Resource manager utilizes the waste heat to generate renewable energy to reduce electricity costs and carbon emissions, which further improves the sustainability of CDC in an efficient manner.

g) Cooling Management Model: A Water based Cooling Management Model [38] is used in terms of Datacenter Cooling System (DCS) Efficiency or cooling effectiveness and it is defined as the following [Eq. 21]:

$$
\begin{aligned}
& \text { DCS Efficiency }=\alpha \frac{\text { Heat }_{\text {Removed }}(t)}{E N C N_{\text {Cooling }}} \\
& \alpha=\text { Temp }_{\text {ExhaustingAir }}-T e m p_{\text {OutsideAir }}
\end{aligned}
$$

Heat $_{\text {Removed }}(t)$ is calculated as the heat absorbed by the heat pump per unit time $t$ and $E N C N_{\text {Cooling }}$ is work done by the cooling devices (compressor, air conditioner and fan) of the heat pump to transfer the thermal energy. Where $\alpha$ is weight to prioritize components of the DCS Efficiency and it is the temperature difference between outside air temperature and the temperature of the (hot) exhausting air of CRAC model [34] as specified in [Eq. 22]. Outside air temperature is the temperature of data center room [64] [65] [66]. The exhausting air is exhausted from server rack, which contains server fans, air conditioners and compressors for smooth functioning of CDC [38]. Different from the outside air cooling, the chiller cooling effectiveness does not change much with temperature and the variation over different IT load is much smaller than that under outside air cooling.

\section{Resource Provisioning and Scheduling}

It is very challenging to schedule provisioned resources for workload execution and maintain reliability and sustainability of cloud service simultaneously [6] [52]. Cloud resource scheduling is a tedious task due to the problem of finding the best match of resource-workload pair based on the user QoS requirements [31] [39] [46]. The problem can be expressed as: mapping a set of independent cloud workloads $\left\{w_{1}, w_{2}, w_{3}, \ldots, w_{m}\right\}$ to a set of heterogeneous and dynamic resources $\left\{r_{1}, r_{2}, r_{3}, \ldots, r_{n}\right\}$ has been taken. For continuous problem, $\mathrm{R}=\left\{r_{k} \mid 1 \leq \mathrm{k} \leq \mathrm{n}\right\}$ is the collection of resources and $n$ is the total number of resources. $\mathrm{W}=\left\{w_{i} \mid 1 \leq \mathrm{i} \leq \mathrm{m}\right\}$ is the collection of cloud workloads and $m$ is the total number of cloud workloads. Figure 2 shows the resource provisioning and scheduling mechanism for execution of user workloads, which determines the most suited resources for a given workload. CRUZE operates by performing the following steps: 1) analyzes workload characteristics with respective QoS requirements, 2) categorizes workload based on their common QoS requirements, 3) provisions cloud resources for categorized workloads and 4) schedule the provisioned resources for workload execution. 


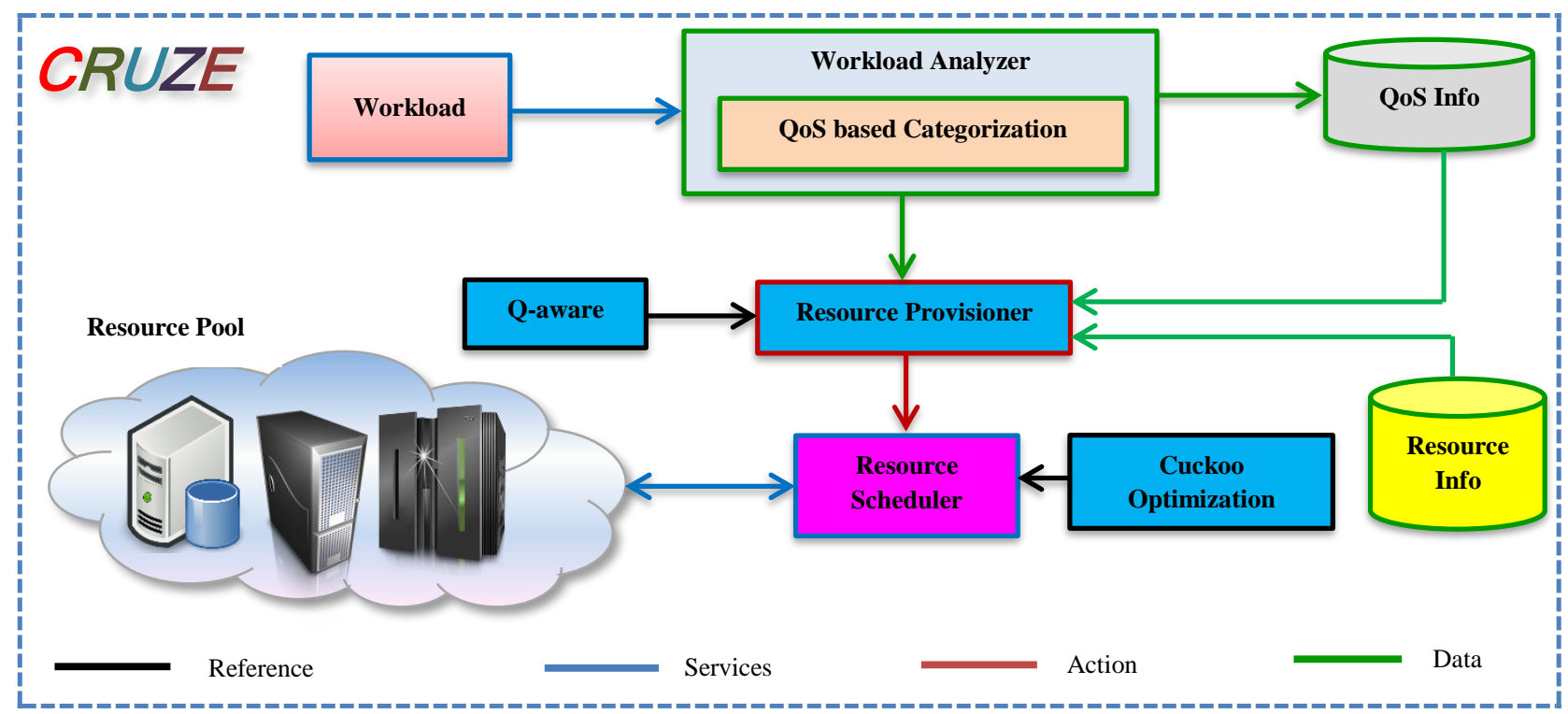

Figure 2: Architecture of Resource Management in CRUZE

\subsection{Clustering of Workloads}

Table 2 lists the various types of workload and their QoS requirements [39] [46], which are considered for this research work.

Table 2: Cloud Workloads and their Key QoS Requirements

\begin{tabular}{|l|l|}
\hline \multicolumn{1}{|c|}{ Workload Name } & \multicolumn{1}{c|}{ QoS Requirements } \\
\hline Technological Computing & Computing capacity \\
\hline Websites & High availability, High network bandwidth and Reliable storage \\
\hline E-Commerce & Customizability and Variable computing load \\
\hline Graphics Oriented & Visibility, Data backup, Latency and Network bandwidth \\
\hline Backup and Storage Services & Persistence and Reliability \\
\hline Endeavour Software & Correctness, High availability and Security \\
\hline Productivity Applications & Security, Data backup, Latency and Network bandwidth \\
\hline Critical Internet Applications & Usability, Serviceability and High availability \\
\hline Mobile Computing Services & Portability, Reliability and High availability \\
\hline Software/Project Development and Testing & Testing time, Flexibility and User self-service rate \\
\hline Central Financial Services & Integrity, Changeability, High availability and Security \\
\hline Online Transaction Processing & Usability, Internet accessibility, High availability and Security \\
\hline Performance Testing & SLA Violation Rate, Resource Utilization, Energy, Cost and Time \\
\hline
\end{tabular}

Further, k-means based clustering algorithm is used for clustering the workloads for execution on different set of resources because k-means clustering has been demonstrating to be an effective means for cloud workload categorization [49]. The process of workload clustering using k-means clustering algorithm has been described in previous research work in detail [48]. Final set of workloads are shown in Table 3.

Table 3: K-Means based Clustering of Workloads

\begin{tabular}{|c|c|c|}
\hline Cluster & Cluster Name & Workloads \\
\hline C4 & Administration & $\begin{array}{c}\text { Graphics Oriented, Software/Project Development and Testing, Productivity Applications, } \\
\text { Central Financial Services, Online Transaction Processing and Endeavour Software }\end{array}$ \\
\hline C3 & Communication & Mobile Computing Services, Critical Internet Applications and Websites \\
\hline C2 & Storage & Backup and Storage Services and E-commerce \\
\hline C1 & Compute & Performance Testing and Technical Computing \\
\hline
\end{tabular}

\subsection{Resource Provisioning}

The resources are provisioned for clustered workload using a resource provisioning technique i.e. Q-aware [31] based on the requirement of workloads of different clusters as described in Table 3. After the provisioning of resources, 
workloads are submitted to resource scheduler. Then, the resource scheduler will ask to submit the workload for resources provisioned. After this, the resource scheduler returns results to the corresponding cloud user, which contains the resource information [31].

\subsection{Cuckoo Optimization based Resource Scheduling Algorithm}

Our proposed scheduling algorithm attempts to minimize overall cloud energy consumption whilst maximizing system reliability. Attaining these two objectives together is typically considered a trade-off; consolidating VMs onto fewer active servers minimizes system energy consumption, server failure can affect multiple VMs and reduce system reliability. In contrast, increasing the number of VM replicas maximizes system reliability, however also incurs additional energy costs due to greater computation requirements and active servers. To overcome this impact, a tradeoff between energy consumption and reliability is required to provide cost-efficient cloud services. Specifically, whilst Dynamic Voltage and Frequency Scaling (DVFS) based energy management techniques can reduce energy consumption, response time and service delay are increased due to the switching of resources between high scaling and low scaling modes. Furthermore, reliability of the system component is also affected by excessive turning on/off servers. Power modulation decreases the reliability of server components such as storage devices, memory etc. Therefore, there is a need of new energy-aware resource management techniques to reduce power consumption whilst incurring minimal impact upon cloud service reliability [5].

Cuckoo Optimization (CO) algorithm is a based resource scheduling technique is designed for execution of user workload considering both energy consumption and reliability. The goal of an objective function is to minimize system energy consumption and maximize server reliability simultaneously for finishing all $\mathrm{n}$ workloads. We define fitness function $(F)$ in terms of energy consumption and reliability as specified in [Eq. 23].

$$
F=\theta E_{\text {consumption }}+\delta R_{\text {service }}
$$

where $0 \leq \theta<1$ and $0 \leq \delta<1$ are weights to prioritize components of the fitness function. Energy consumption ( $\left.E_{\text {consumption }}\right)$ and Reliability $\left(R_{\text {service }}\right)$ is calculated using [Eq. 10] and [Eq. 11] respectively. This objective function successfully captures the compromise among QoS parameters as specified in [Eq. 23]. Cuckoo Optimization (CO) algorithm is motivated by the life of the cuckoo bird [28] as it adapts the features of a cuckoo and process of laying eggs. $\mathrm{CO}$ algorithm has both local and global search abilities and the performance of the $\mathrm{CO}$ algorithm has been demonstrated to be more effective in comparison to PSO and ACO in terms of accuracy, speed and convergence [51] for solving optimization problems such as batch process scheduling and job scheduling [28] [29]. The mapping and execution of the workloads on suitable cloud resources is recognized to be an NP-complete problem and there is a need for novel algorithm for resource scheduling with maximum reliability and sustainability of cloud services [6]. We have selected $\mathrm{CO}$ algorithm for scheduling of provisioned resources due to following reasons: a) capability to schedule resources for workload execution automatically, b) relatively straight forward integration with traditional optimization techniques, and c) easy modification in a dynamic cloud environment. Resource Utilization is a ratio of execution time of a workload executed by a particular resource to total uptime of that resource and it is specified in [Eq. 24]. The total uptime of resource is the amount of time that a resource from a resource set is available for execution of workloads.

$$
R_{U}=\sum_{i=1}^{n}\left(\frac{\text { execution time of a workload executed on } i^{\text {th }} \text { resource }}{\text { total uptime of } i^{\text {th }} \text { resource }}\right)
$$

where $n$ is the no. of resources. A resource set consist of number of instances. [Eq. 25] shows $i^{\text {th }}$ resource $\left(\mathrm{R}_{i}\right)$ contains instances $(I)$ :

$$
\mathrm{R}_{i}=\left[I_{i 1}, I_{i 2}, \ldots \ldots \ldots I_{i X}\right] \text {, where } I_{i 1}, I_{i 2}, \ldots \ldots \ldots I_{i X} \text { are instances of } i^{\text {th }} \text { resource and } \mathrm{x} \leq 50 .
$$

The value of resource utilization depends on the number of instances of that resource are using to execute the workload. Resource utilization for $i^{\text {th }}$ resource $\left(\mathrm{R}_{i}\right)$ is shown in [Eq. 26].

$$
R_{U} i=\frac{\sum_{a=1}^{x}\left(\text { Execution Time of Workload on } a^{\text {th }} \text { resource }\right)}{\sum_{a=1}^{x}\left(\text { total uptime of } a^{\text {th }} \text { resource }\right)}
$$


where $x$ is the number of instances and we have assumed the value of $\mathrm{x} \leq 50$ for this research work. Figure 3 shows the flowchart of $\mathrm{CO}$ algorithm based resource scheduling.

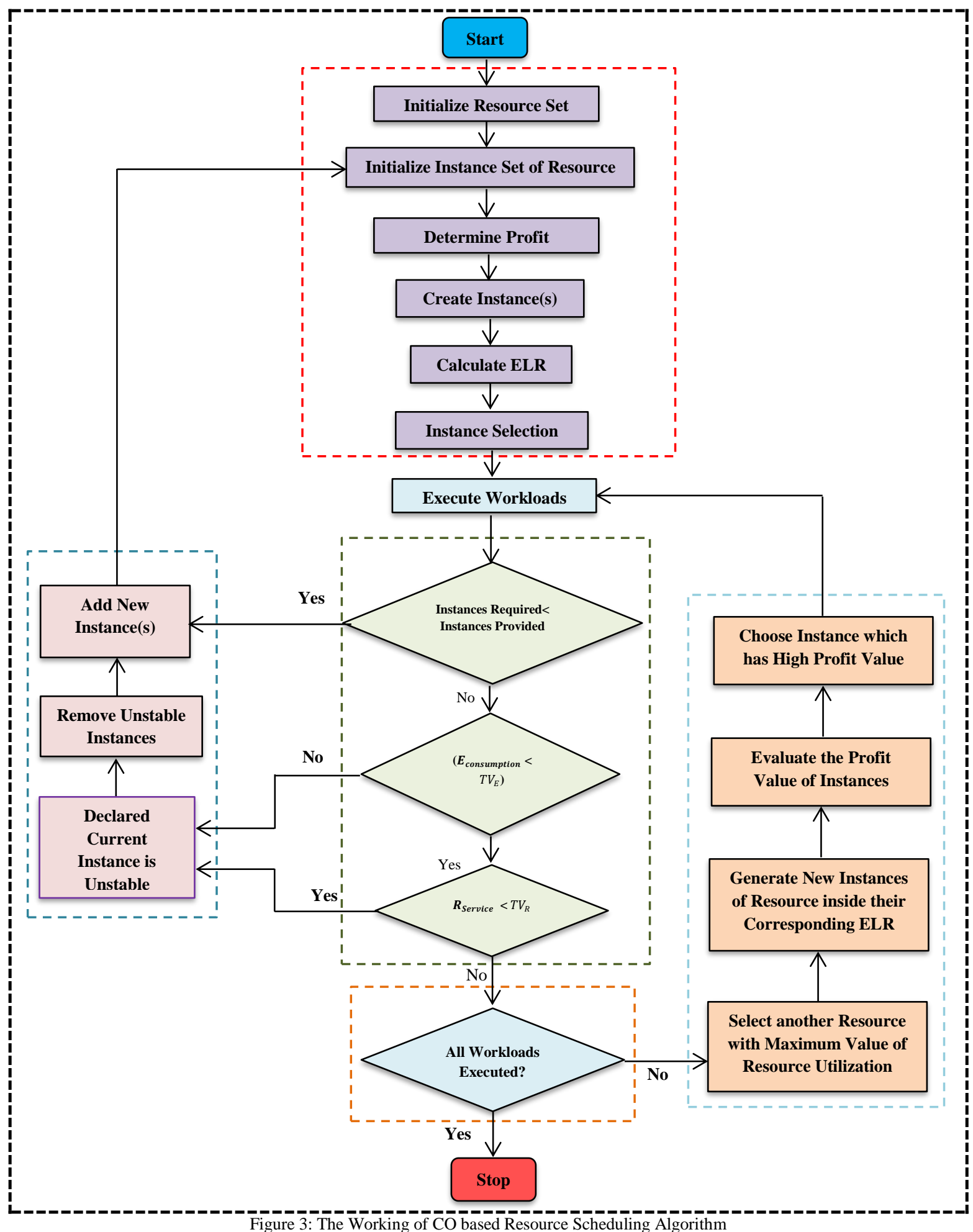

Similar to the other evolutionary algorithms, this algorithm starts with an initial population. In this research work, we have modified the $\mathrm{CO}$ algorithm based on the requirements of cloud resource scheduling. We have considered as Mature cuckoos (existing provisioned resources) and their Eggs (new instances). Based on different values of resource 
utilization $\left(R_{U}\right)$, initial population is considered as a resource set and different resources are sorted in decreasing order ( $R_{U_{1}} \geq R_{U_{2}} \geq \cdots \geq R_{U_{n}}$ ). There are new instances of those resources to be added to a specific resource for future execution of workloads and these instances will become part of resource after producing required performance ( $\mathrm{E}_{\text {consumpton }}<T V_{E} \& \& \mathrm{R}_{\text {Service }}>T V_{R}$ ), where $T V_{E}$ is a threshold value for energy and $T V_{R}$ is a threshold value for reliability (which are decided based on the historic data of past execution of workloads [31] [46]). The more number of instances are adding to a resource pool, the more profit is gained (in terms of resource utilization). Therefore, the improvement in resource utilization will be the definition that $\mathrm{CO}$ algorithm intends to optimize.

The main objective of $\mathrm{CO}$ the algorithm in this research work is to increase utilization of resources by selecting best resource based on their fitness value. Cuckoo search finds the most suitable resource to create more instances in order to maximize their resource utilization. After new instances performing as required, they come together to make new resources. Each instance has its resource to execute workloads. The best instance among all the instances will be the destination for the workloads for their execution. Then they move toward this best resource. They will inhabit near the best resource. Considering the number of instances each resource has and the resource's distance to the goal point (best resource), some range of resource (in terms of Egg Laying Radius (ELR)) is dedicated to it, and is calculated using [Eq. 33]. There is no obvious metric on the space of resource sets, as opposed to $n$-dimensional space. The next step is that a resource begins to create instances in a stochastic manner inside the resource range, defined by the value of ELR. This process lasts until the best resource with extreme value of profit (in terms of resource utilization) is obtained and most of the instances of resource are gathered around the same position.

The following are important functions of $\mathrm{CO}$ based resource scheduling algorithm:

a) Initialize Resource Set: Cuckoo Habitat as a resource set $\left(\right.$ Resource $\left._{S e t}\right)$ is considered in CO based resource scheduling algorithm. The resource set is an array of $1 \times q_{v a r}$ in $q_{v a r}$-dimensional optimization problem, the resource set is demonstrated as follows [Eq. 26]. Resource set contains different number of resources.

$$
\text { Resource }_{\text {Set }}=\left[R_{1}, R_{2}, \ldots \ldots \ldots R_{q_{v a r}}\right] \text {, where } R_{1}, R_{2}, \ldots \ldots \ldots R_{q_{v a r}} \text { are resources }
$$

b) Initialize Instance Set of Resource: Furthermore, every resource contains instances (I) as shown in [Eq. 28].

$$
\mathrm{R}_{q_{v a r}}=\left[I_{q_{v a r} 1}, I_{q_{v a r} 2}, \ldots \ldots \ldots I_{q_{v a r} X}\right] \text {, where } I_{q_{v a r} 1}, I_{q_{v a r} 2}, \ldots \ldots \ldots I_{q_{v a r} X} \text { are instances and } \mathrm{x} \leq 50
$$

where $x$ is the number of instances and we have assumed the value of $\mathrm{x} \leq 50$ for this research work. $I_{q_{v a r} i} \in\{0$, $1\}$, where $1 \leq i \leq 50$. The value 1 state that the particular instance is initialized and 0 represent the elimination of that instance from the final set.

c) Determine Profit: The profit of a resource set is obtained by evaluation of profit function at a resource set $\left(R_{1}, R_{2}, \ldots \ldots \ldots R_{m}\right)$. So, profit function is shown in [Eq. 29]:

$$
\begin{gathered}
\text { Profit }=R_{U}\left(\text { Resource }_{\text {Set }}\right)=R_{U}\left(R_{1}, R_{2}, \ldots \ldots \ldots R_{q_{v a r}}\right) \\
\text { Profit }=R_{U}\left(\left(I_{11}, I_{12}, \ldots \ldots \ldots I_{1 X}\right),\left(I_{21}, I_{22}, \ldots \ldots \ldots I_{2 X}\right), \ldots \ldots \ldots \ldots \ldots\left(I_{q_{v a r} 1}, I_{q_{v a r}}, \ldots \ldots \ldots I_{q_{v a r} X}\right)\right)
\end{gathered}
$$

Maximize the profit in terms of cost $\left(-c_{t}\right)$ for cost optimization of resource scheduling. To apply the CO algorithm to solve the minimization problems, it is sufficient to multiply the minus sign by cost function. A negative sign means that an improvement in the respective resource utilization results in a reduced cost. If the resource utilization reduces, then it results in an increased cost (because negative times negative results in a positive). The magnitude of the change is given by the value of the cost. The sign gives the direction of the change.

$$
\begin{gathered}
\text { Profit }=-\operatorname{Cost}\left(\text { Resource }_{\text {Set }}\right)=-c_{t}\left(R_{1}, R_{2}, \ldots \ldots \ldots R_{q_{v a r}}\right) \\
\text { Profit }=-c_{t}\left(\left(I_{11}, I_{12}, \ldots \ldots \ldots I_{1 X}\right),\left(I_{21}, I_{22}, \ldots \ldots \ldots I_{2 X}\right), \ldots \ldots \ldots \ldots \ldots\left(I_{q_{v a r} 1}, I_{q_{v a r}}, \ldots \ldots \ldots I_{q_{v a r} X}\right)\right)
\end{gathered}
$$

To begin the optimization algorithm, a candidate Resource $_{\text {set }}$ matrix of size $q_{p o p} \times q_{v a r}$ is created, where $q_{p o p}$ is the value of an initial population considered in a resource set. Then some randomly produced number of instances is supposed for each of these initial resource sets. 
d) Create Instance(s): In this research work, each resource creates 1 to 50 instances. These values are used as the upper and lower limits of instance creation to each resource set at different iterations. In CO algorithm, instances are creating within a maximum distance from their resource set and this maximum range is known as Egg Laying Radius (ELR). Based on the ELR value, every resource generates instances as shown in Figure 4. In a resource, there are two types of instances: stable and unstable. Stable instances are those which have maximum value in terms of resource utilization and fitness value [Eq. 23]. Further, stable instance has also a capability to execute at least certain number of workloads. Otherwise, it is called as unstable instance.

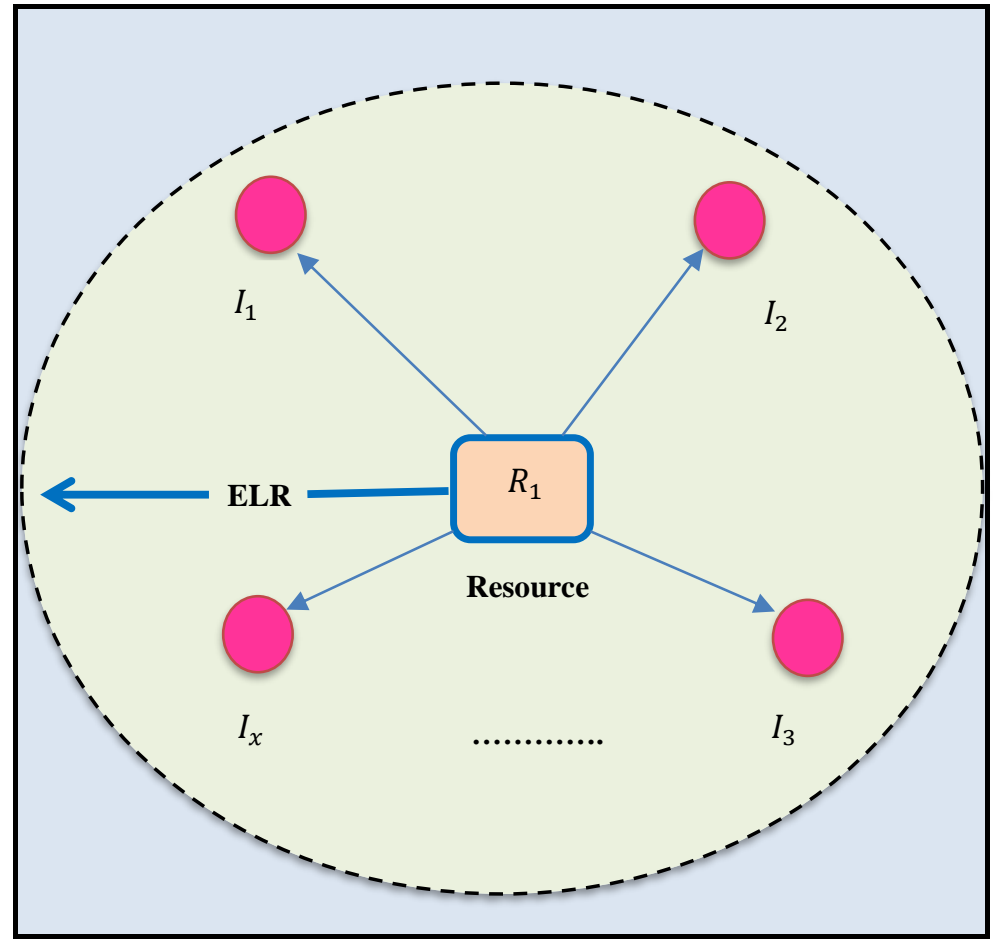

Figure 4: Resource creating their Instances within the Region defined by ELR

e) Calculate ELR: ELR is defined as the ratio of number of instances of a current resource are executing a particular workload to the total number of active instances of that resource and it is described in Eq. [33].

$$
\mathrm{ELR}=\mu \times\left(\frac{\text { number of instances of } i^{\text {th }} \text { resource executing a particular workload }}{\text { total number of active instances of } i^{\text {th }} \text { resource }}\right) \times\left(\text { var }_{U}-\text { var }_{L}\right)
$$

In an optimization problem with upper limit of $\operatorname{var}_{U}$ and lower limit of $v^{2} r_{L}$ for variables, each resource set has an ELR, which is proportional to the total number of instances of a resource set and also variable (var) limits of $\operatorname{var}_{U}$ and $\operatorname{var}_{L} \cdot \mu$ is an integer, supposed to handle maximum value of ELR.

f) Instance Selection: $\mathrm{CO}$ based resource scheduling algorithm i) finds the number of unstable instances, ii) selects the resource with minimum value of unstable instances, and iii) create instances of selected resource to execute set of workloads. Instance is selected based on its Fitness Value (F), calculated using [Eq. 33] and start execution of workloads.

g) Monitor Performance: The performance of workload execution is monitored continuously and checks the instance requirement (whether the provided instances are sufficient for execution of current set of cloud workloads). The more number of instances are provided to continue execution if provided instances are less than required instances. It calculates the value of energy consumption and reliability. The value of energy consumption ( $\left.\mathrm{E}_{\text {consumption }}\right)$ associated with it should be less than Threshold Value $\left(T V_{E}\right)$ and the value of reliability $\left(\mathrm{R}_{\text {Service }}\right)$ associated with it should be more than Threshold Value $\left(T V_{R}\right)$ for successful execution of workloads. Otherwise, it declares the current instance as an unstable, eliminates unstable instance and add new instance using following 
steps: a) select another resource with maximum value of resource utilization, b) generate new instances of resource inside their corresponding ELR, c) evaluate the profit value of instances and d) choose instance which has higher profit value. The performance is monitored continuously until all the workloads are not executed.

The main steps of CO based resource scheduling algorithm are presented as a pseudo-code in [ALGORITHM 1].

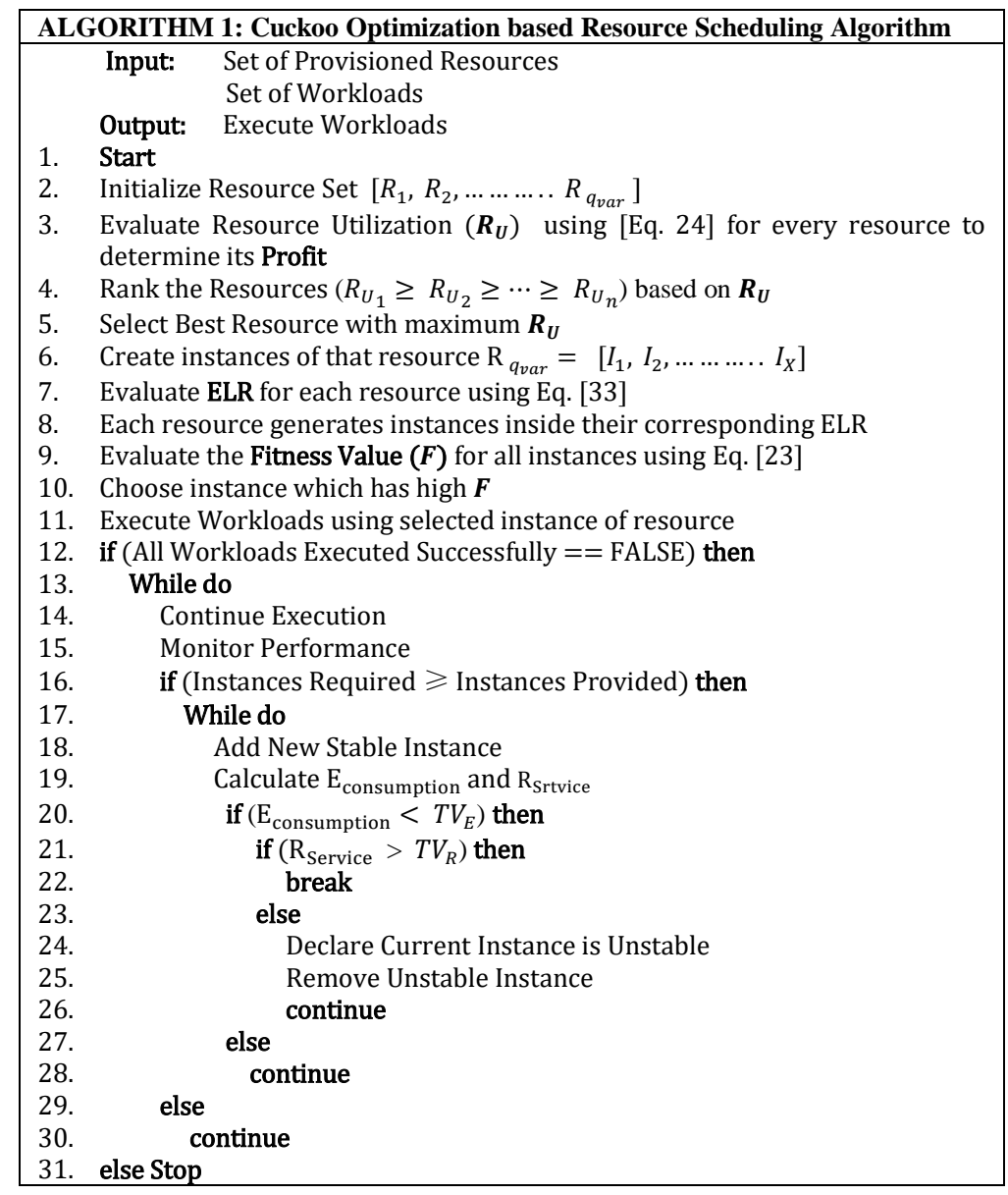

Initially, provisioned resources as an input for scheduling of resources to execute cloud workloads, and both workload and resource set contains integer values for our technique. Firstly, $\mathrm{CO}$ based resource scheduling algorithm initializes the resources. Further, it evaluates the resource utilization of all the resources using [Eq. 24] to determine the profit and sorts the resources in decreasing order $\left(R_{U_{1}} \geq R_{U_{2}} \geq \cdots \geq R_{U_{n}}\right)$ based on their value of resource utilization. Then, it selects the best resource based on the maximum value of resource utilization $\left(\mathrm{R}_{\mathrm{U}}\right)$. Further, it creates some number of instances $\left[I_{1}, I_{2}, \ldots \ldots \ldots I_{X}\right]$ for every resource and evaluates the value of the ELR for each resource using [Eq. 33]. Moreover, each resource generates instances inside their corresponding ELR and evaluate the Fitness Value (F) for all instances using Eq. [23] and determine the best individual with the best fitness value (which has maximum value of $R_{U}$ and the value of energy consumption associated with it is less than a threshold value and the value of reliability is more than its threshold value).Further, $\mathrm{CO}$ based resource scheduling algorithm starts execution of workloads and it checks the execution status of workloads. If all the workloads are executed successfully then execution stops otherwise it continues execution of workloads. The performance is monitored continuously during execution of cloud workloads. It checks the instance requirement (whether the provided instances are sufficient for execution of current set of cloud workloads). The more number of instances are provided to continue execution if provided instances are less than required instances. It calculates the value of energy consumption and reliability. The value of energy consumption ( $\mathrm{E}_{\text {consumption }}$ ) associated with it should be less than Threshold Value $\left(T V_{E}\right)$ and the value of reliability $\left(\mathrm{R}_{\text {Service }}\right)$ associated with it should be more than Threshold Value $\left(T V_{R}\right)$ for successful execution of workloads. Otherwise, it declares the current instance as an unstable, eliminates unstable instance and add new 
instance using following steps: 1) select another resource with maximum value of resource utilization, 2) generate new instances of resource inside their corresponding ELR, 3) evaluate the profit value of instances and 4) choose instance which has higher profit value. The performance is monitored continuously until all the workloads are not executed.

\section{Performance Evaluation}

We modelled and simulated a cloud environment using CloudSim [30], a prominent cloud computing simulation framework. Figure 5 shows the interaction of different entities for simulation. Table 4 presents the resource configuration of the simulation as we used in our previous research work [39] [46]. We used three Physical Machines (PMs) with different number of virtual nodes (6, 4 and 2) and virtual nodes are further divided into instances called Execution Components (ECs).

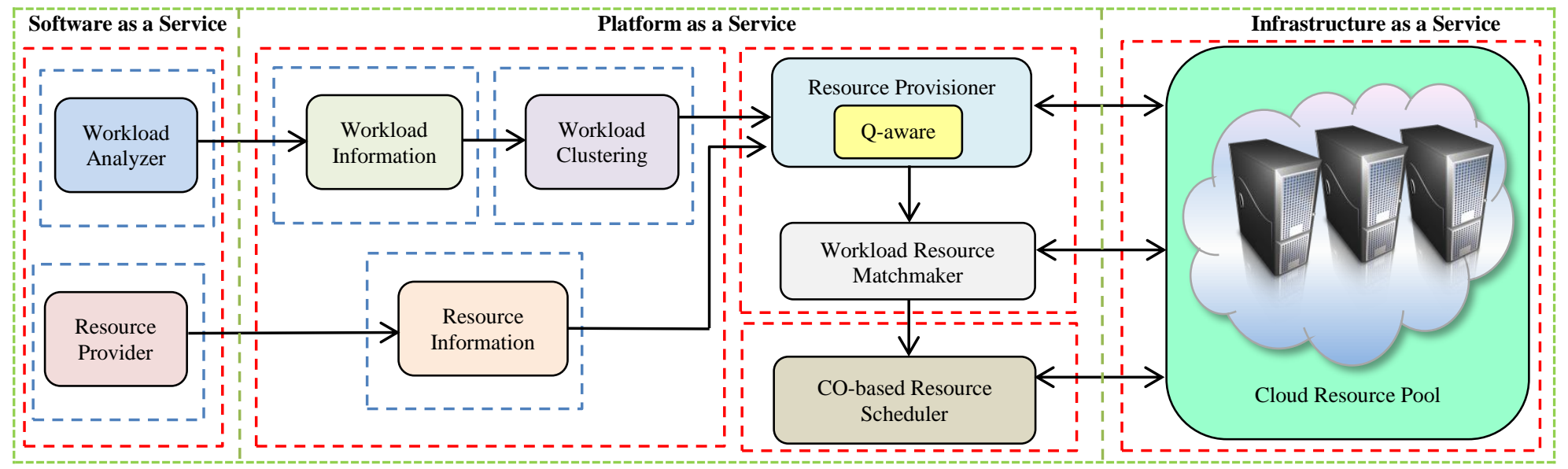

Figure 5: Interaction of various Entities in the Simulated Cloud Environment

Every EC contains their own cost of execution and it is measured with unit (C\$/EC time unit (Sec)). EC measures cost per time unit in Cloud dollars (C\$).

Table 4: Configuration Details

\begin{tabular}{|c|c|c|c|c|c|c|c|}
\hline Resource_Id & Configuration & Specifications & Core & $\begin{array}{l}\text { Operating } \\
\text { System }\end{array}$ & $\begin{array}{c}\text { Number of } \\
\text { Virtual Nodes }\end{array}$ & $\begin{array}{c}\text { Number } \\
\text { of ECs }\end{array}$ & $\begin{array}{c}\text { Price } \\
\text { (C\$/EC } \\
\text { time unit) }\end{array}$ \\
\hline R1 & $\begin{array}{l}\text { Intel Core } 2 \\
\text { Duo }-2.4 \mathrm{GHz}\end{array}$ & $\begin{array}{c}6 \text { GB RAM and } \\
320 \text { GB HDD }\end{array}$ & 2 & Windows & $\begin{array}{c}6(1 \mathrm{~GB} \text { and } \\
50 \mathrm{~GB})\end{array}$ & 18 & 2 \\
\hline R2 & $\begin{array}{l}\text { Intel Core i5- } \\
2310-2.9 \mathrm{GHz} \\
\end{array}$ & $\begin{array}{c}4 \text { GB RAM and } \\
160 \text { GB HDD }\end{array}$ & 2 & Linux & $\begin{array}{c}4(1 \mathrm{~GB} \text { and } \\
40 \mathrm{~GB})\end{array}$ & 12 & 3 \\
\hline R3 & $\begin{array}{l}\text { Intel XEON E } \\
52407-2.2 \mathrm{GHz}\end{array}$ & $\begin{array}{c}2 \text { GB RAM and } \\
160 \text { GB HDD }\end{array}$ & 2 & Linux & $\begin{array}{c}2(1 \mathrm{~GB} \text { and } \\
60 \mathrm{~GB})\end{array}$ & 6 & 4 \\
\hline
\end{tabular}

We have integrated temperature and cooling management model [34], renewable energy model [35], waste heat management model [36], security manager [47] and Fault Injection Module (FIM-SIM) [45] to the CloudSim toolkit for simulation as shown in Figure 6. We have integrated FIM-SIM [45] for fault management in CloudSim toolkit to simulate failures (VM creation failures and host failures) as discussed in Case-1 of Section 5.3. The detailed description about experimental setup is given in previous research work [39] [46].

For the execution of workloads in our experiments, we have chosen varied computational settings on top of heterogeneous resources. The variety comes in the number of cores at the CPU-level, the page levels of the main memory, switches at the network level and disk space at the storage level [30] [69] [70]. Cores is the number of Processing Element's (PE) required by the Cloudlet. Table 5 shows the simulation parameters utilized in the various experiments undertaken by this research work, also as identified from the existing empirical studies and literature such as fault management [6] [39] [46], application's QoS [39] [44] [47] [46] [67], capacity planning [33] [65], energy management [6] [7] [44] [67], waste heat utilization [36] [65], renewable energy [35] [38], virtualization [6] [7] [67], 
thermal-aware scheduling [34] [66] [68] and cooling management [38] [65] [66] [68]. Experimental setup incorporated CloudSim to produce and retrieve simulation results.

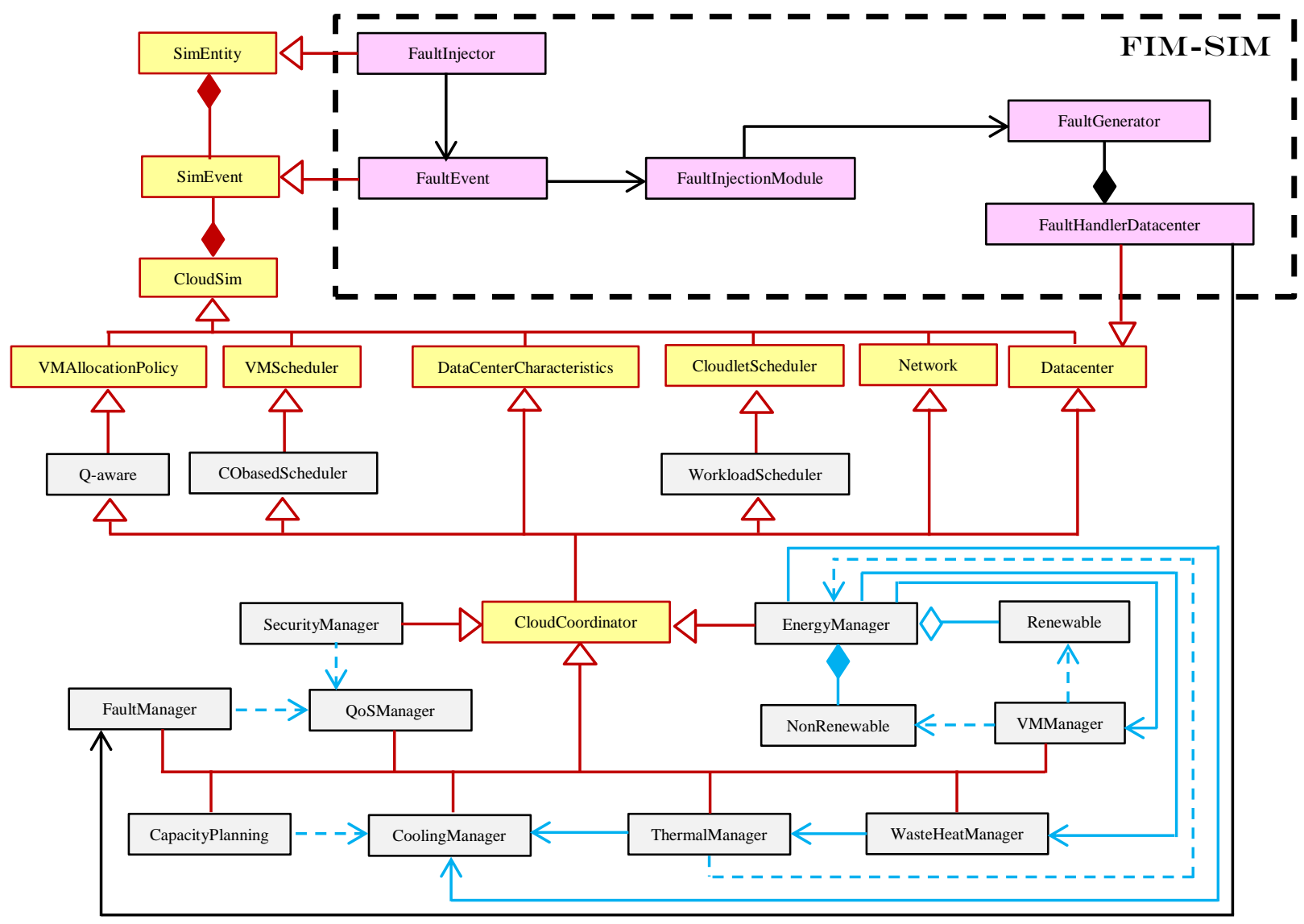

\subsection{Workload}

Figure 6: Design Description using Class Diagram

For performance evaluation, we have selected four different types of cloud workload from every cluster of workloads as given in Table 3. Table 6 shows the different cloud workloads, which are considered to evaluate the performance of CRUZE. To find the experiment statistics, 500-3000 different workloads are executed. CRUZE processes different workloads using the different number of resources to evaluate its performance with different resource configuration. We selected the Poisson Distribution [31] for workload submission in this research work due to following reasons: 1) evaluating the performance of workload execution for specific interval of time and 2) every workload is independent of all other workloads (number of workloads are arriving in first hour is independent of the number of workloads arriving in any other hour).

Table 5: Simulation Parameters and their Values

\begin{tabular}{|l|c|}
\hline \multicolumn{1}{|c|}{ Parameter } & Value \\
\hline Number of VMs (n) & 36 \\
\hline Number of Cloudlets (Workloads) & 3000 \\
\hline Bandwidth & $1000-3000 \mathrm{~B} / \mathrm{S}$ \\
\hline CPU MIPS & 2000 \\
\hline Size of Cloud Workload & $10000+(10 \%-30 \%) \mathrm{MB}$ \\
\hline Number of PEs per machine & 1 \\
\hline PE ratings & $100-4000 \mathrm{MIPS}$ \\
\hline Cost per Cloud Workload & $3 \mathrm{C} \$-5 \mathrm{C} \$$ \\
\hline Memory Size & $2048-12576 \mathrm{MB}$ \\
\hline File size & $300+(15 \%-40 \%) \mathrm{MB}$ \\
\hline Cloud Workload output size & $300+(15 \%-50 \%) \mathrm{MB}$ \\
\hline CPU Temperature & $10-27^{\circ} \mathrm{C}$ \\
\hline
\end{tabular}




\begin{tabular}{|l|c|}
\hline Inlet Temperature & $15-40{ }^{\circ} \mathrm{C}$ \\
\hline$W_{m}=$ mass flow rate of the water entering the circulation system & $0.08-0.024 \mathrm{~kg} / \mathrm{s}$ \\
\hline$W_{s}=$ mass flow rate of the steam generated in the circulation system & $0.03-0.134 \mathrm{~kg} / \mathrm{s}$ \\
\hline Power $(\mathrm{KW})$ & $108-273 \mathrm{KW}$ \\
\hline Latency & $20-90 \mathrm{Seconds}$ \\
\hline Heat $t_{\text {Removed }}$ & $100-1,000 \mathrm{Joules} / \mathrm{Second}$ \\
\hline Cache Memory Size & $4 \mathrm{MB}-16 \mathrm{MB}$ \\
\hline Energy Reused & $40-85 \%$ \\
\hline Power Consumption by Processor & $130 \mathrm{~W}-240 \mathrm{~W}$ \\
\hline Power Consumption by Cooling Devices & $400 \mathrm{~W}-900 \mathrm{~W}$ \\
\hline Power Consumption by RAM & $10 \mathrm{~W}-30 \mathrm{~W}$ \\
\hline Power Consumption by Storage & $35 \mathrm{~W}-110 \mathrm{~W}$ \\
\hline Power Consumption by Network & $70 \mathrm{~W}-180 \mathrm{~W}$ \\
\hline Power Consumption by Extra Components & $2 \mathrm{~W}-25 \mathrm{~W}$ \\
\hline Equipment cost $\left(E_{i}\right)$ & $4-30 \mathrm{C} \$$ \\
\hline Support contract cost $\left(\mathrm{S}_{i}\right)$ & $5-15 \mathrm{C} \$$ \\
\hline Administrative costs $\left(\mathrm{A}_{i}\right)$ & $15-50 \mathrm{C} \$$ \\
\hline Power cost per month $\left(\mathrm{P}_{i}\right)$ & $12-30 \mathrm{C} \$$ \\
\hline Rack cost per month $\left(\mathrm{R}_{i}\right)$ & $3-12 \mathrm{C} \$$ \\
\hline Communication cost $\left(\mathrm{C}_{i}\right)$ & $2-17 \mathrm{C} \$$ \\
\hline
\end{tabular}

CRUZE also maintains the details of every executed workload and stores into workload database, which can be used to test the efficiency of CRUZE in future. For experimental results, we executed four different workloads [(i) Storage and Backup Data, (ii) Websites, (iii) Performance Testing and (iv) Software Development and Testing] with the same experimental setup.

Table 6: Details of Cloud Workloads

\begin{tabular}{|c|c|l|}
\hline Workload & Cluster & \multicolumn{1}{c|}{ Description } \\
\hline Performance Testing & Compute (C1) & $\begin{array}{l}\text { CRUZE processes and converts an image file (713 MB) to PNG format from JPG format. } \\
\text { The change of a one JPG file into PNG is taken as a workload (in the form of Cloudlet). }\end{array}$ \\
\hline $\begin{array}{c}\text { Storage and Backup } \\
\text { Data }\end{array}$ & Storage (C2) & $\begin{array}{l}\text { Store a huge chunk of data (5 TB) and generates a backup of data is considered as a } \\
\text { workload. }\end{array}$ \\
\hline Websites & Communication (C3) & $\begin{array}{l}\text { A large number of users are accessing a website of university during Admission Period is } \\
\text { considered as a workload. }\end{array}$ \\
\hline $\begin{array}{c}\text { Software Development } \\
\text { and Testing }\end{array}$ & Administration (C4) & $\begin{array}{l}\text { Development and testing of an Agri-Info Software to find out the productivity of a crop is } \\
\text { considered as a workload [32]. }\end{array}$ \\
\hline
\end{tabular}

\subsection{Baseline Resource Scheduling Approaches}

In order to evaluate our approach, we have selected three state-of-the-art resource scheduling approaches from the literature (as discussed in Section 2). We have selected most relevant and recent similar work such as HMRM [13], CSRE [16] and CSMH [27] to evaluate the performance of our proposed approach. The other reasons of selection of these existing scheduling approaches are: HMRM [13] manages cloud resources holistically by focusing on the energy consumption and CSRE [16] executes workloads by improving the reliability of cloud service, while CSMH [27] schedule resources in an energy-efficient manner using Cuckoo search meta-heuristic algorithm.

1) HMRM [13]: Holistic Model for Resource Management (HMRM) approach is designed for virtual cloud environment to reduce energy consumption of different components of cloud datacenters such as storage and network without focusing on memory, processors, cooling systems. HMRM executes only homogeneous cloud workloads.

2) CSRE [16]: Cloud Service Reliability Enhancement (CSRE) approach is developed to improve the storage and network resource utilization during execution of workloads. CSRE uses service checkpoint to store the state of all the VMs, which are currently processing user workloads. Further, a node failure predicator is developed to reduce the network resource consumption. CSRE executes only homogeneous workloads and considers only two types of resources such as storage and network without focusing on memory, processors, cooling systems.

3) CSMH [27]: Cuckoo Search Meta-Heuristic (CSMH) algorithm based resource scheduling approach is designed to optimize the energy consumption of cloud resources (processors only) for execution of homogeneous workloads without focusing on other resources such as networks, memory, storage, cooling systems. 
Our proposed approach (CRUZE) focuses on holistic management of all resources (including servers, networks, memory, storage, cooling systems) to provide reliable as well as sustainable cloud services simultaneously, which schedules the provisioned resources using evolutionary algorithm (Cuckoo Optimization) for the execution of clustered and heterogeneous workloads within their specified deadline, budget and other important QoS parameters.

\subsection{Experimental Results}

All the experiments utilized four different workloads described in Table 6. The various parameters are used to evaluate the performance of proposed approach for holistic resource management, which comprises of different categories such as fault management, application's QoS, capacity planning, energy management, waste heat utilization, renewable energy, virtualization, thermal-aware scheduling and cooling management. The temporal evaluations are conducted in a time period of 12 hours with 3000 workloads submitted. The performance of CRUZE is evaluated using following different test cases:

Case 1 - Fault Management: We have evaluated the performance of CRUZE in terms of reliability and fault detection rate for fault tolerance and used [Eq. 11] to measure the value of reliability. Fault detection rate is defined as the ratio of number of faults/failures (hardware, software, network) detected to the total number of faults/failures in the system [46]. Fault Detection Rate (FDR) is calculated using [Eq. 34].

$$
\text { FDR }=\frac{\text { Number of Faults Detected }}{\text { Total number of Faults }}
$$

Faults can be a network, software or hardware, which is detected based on the violation of SLA. The Software faults/failures can be occurred due to following reasons: 1) lesser storage space, 2) resource unavailability, 3) deadlocks and 4) unhandled exceptions. The reasons of hardware faults/failures can be problems in hardware parts such as hard disk, primary memory and processor. Network error can be breakage of network, scalability or physical damage.

FIM-SIM: We have integrated Fault Injection Module (FIM-SIM) [45] [46] to inject faults automatically to test the reliability of CRUZE as shown in Figure 6. FIM-SIM is working based on event-driven models and injects faults into the CloudSim [30] using different statistical distributions at runtime. A Weibull Distribution is used in order to model failures characteristics when injecting faults [46]. We injected three types of faults: VM creation failures, host failures (Processing Elements failure and memory failure) and high-level failures like cloudlets failures (which are caused by any networking problem that CloudSim [30] cannot handle). The entities in CloudSim [30] communicate through messages. Since host and VM are static entities, each change in their state should be realized by the datacenter. The broker, based on the simulation configuration (number of cloudlets and their specification) will request the VM creation, cloudlet scheduling and it will wait to be informed by the datacenter when the cloudlets completion is realized. We have simulated VM creation failures, host failures (hardware failure) and cloudlets failures (network failure) by creating fault injector thread, which sends the failure event based on the following command: sendNow(dataCenter.getId(), FaultEventTags.HOST_FAILURE, host); and it generates the events based on statistical distribution using Weibull Distribution [46]. The Fault Tolerance Module is extending the CloudSim core functions (see Figure 6) with three entities (FaultInjector, FaultEvent and FaultHandlerDatacenter) as described in Table 7.

Table 7: Entitles of FIM-SIM and their functionalities

\begin{tabular}{|c|c|c|}
\hline njector & FaultEvent & FaultHandlerDatacenter \\
\hline $\begin{array}{l}\text { - } \\
\text { Extends the SimEntity class } \\
\text { starts at simulation startup along with } \\
\text { the other entities from the system } \\
\text { Responsible for inserting fault events at } \\
\text { random moments of time } \\
\text { The random generation of moments of } \\
\text { time is based on a statistical distribution } \\
\text { (We used Weibull Distribution [46] for } \\
\text { this research work.) }\end{array}$ & $\begin{array}{l}\text { - } \\
\text { Extends the SimEvent class } \\
\text { Describes a fault event: source, } \\
\text { destination, time and type; - tag type: } \\
\text { HOST FAILURE, CLOUDLET } \\
\text { FAILURE, CREATE VM FAILURE } \\
\text { - Created in the Fault Injection Module. }\end{array}$ & $\begin{array}{l}\text { - } \\
\text { - } \quad \text { procends the Datacenter class } \\
\text { FaultGenerator } \\
\text { It updates the cloudlet execution/status } \\
\text { according to the fault event type } \\
\text { handles VM migration; - since host and } \\
\text { VM are static entities, all its state } \\
\text { modification should be processed by the } \\
\text { datacenter. }\end{array}$ \\
\hline
\end{tabular}


CRUZE uses the concept of Carburizer [37] [46] to perform process of hardware hardening, which reduces the frequency of faults/failures. CRUZE replaces the new driver (harden driver) with original in case of fault and update the database regarding new faults to avoid future faults, which improves the fault detection rate in CRUZE as compared to HMRM, CSRE and CSMH. Figure 7 (a) shows the variation of fault detection rate for CRUZE, HMRM, CSRE and CSMH. Fault detection rate is decreasing as number of workloads increases for CRUZE, HMRM, CSRE and CSMH, but CRUZE performs better than HMRM, CSRE and CSMH. The average value of fault detection rate in CRUZE is $19.99 \%, 21.14 \%$ and $22.45 \%$ more than HMRM, CSRE and CSMH respectively. Dynamic Random-Access Memory (DRAM) provides the Check-pointing mechanism to store the current states of VMs in case of failure [46]. Figure 7 (b) shows the variation of reliability for CRUZE, HMRM, CSRE and CSMH with different number of workloads (500-3000). The average value of reliability in CRUZE is $19.07 \%, 19.75 \%$ and $20.98 \%$ more than HMRM, CSRE and CSMH respectively.

Case 2 - Application QoS: We have considered three performance parameters for application's QoS: execution cost, time and security [37]. Execution cost is defined as the total money that can be spent in one hour to execute the application successfully and execution cost is measured in Cloud Dollars (C\$) [2]. We have used following formula to calculate Execution Cost (C) [Eq. 35].

$$
C=E_{t} \times \text { Price }
$$

where "price" of a resource is calculated using Table 4 and the value of $E_{t}$ is calculated using [Eq. 36]. Figure 7 (c) shows the comparison of CRUZE, HMRM, CSRE and CSMH in terms of execution cost and cost is increasing with increase in number of workloads for CRUZE, HMRM, CSRE and CSMH, but CRUZE consumes less cost as compared to HMRM, CSRE and CSMH. The average value of cost in CRUZE is $14.41 \%, 14.91 \%$ and $15.46 \%$ less than HMRM, CSRE and CSMH respectively.

In resource scheduler, CRUZE considers the impact of other workloads on current workload during execution. CRUZE schedules provisioned resources using Q-aware [37], which clusters the workloads and execute within their specified deadline and budget. Execution time is the amount of time required to execute application successfully and execution time is measured in Seconds [2]. [Eq. 36] is used to calculate Execution Time $\left(E_{t}\right)$.

$$
E_{t}=\sum_{i=1}^{m}\left(\frac{W C_{i}-W S_{i}}{m}\right)+\Delta t_{i}
$$

Where $W C_{i}$ is workload completion time and $W S_{i}$ is workload submission time, $\Delta t_{i}$ is time to restart the node and $m$ is the number of workloads. Figure 7 (d) shows the variation of an execution time with different number of workloads and time is increasing with increase in number of workloads for both CRUZE, HMRM, CSRE and CSMH. The average value of execution time in CRUZE is $9.96 \%, 10.35 \%$ and $12.11 \%$ less than HMRM, CSRE and CSMH respectively because CRUZE tracks the resource states automatically for effective decisions. Security is an ability of the computing system to protect the system from malicious at tacks and measured in terms of Intrusion Detection Rate (IDR) [47]. IDR is described in [Eq. 37], which is the ratio of total number of true positives to the total number of intrusions.

$$
I D R=\frac{\text { Total Number of True Positives }}{\text { Total Number of Intrusions }}
$$

IDR considers the number of detected and blocked attacks. CRUZE deploys security agents on different computing systems, which trace unknown attacks (using an anomaly-based detector) and known attacks (using a signature-based detector). It captures new anomalies based on existing data stored in the central database (SNORT DB). CRUZE captures and detects anomalies using the Intrusion Detection System and labels it as anomalous or normal traffic data by comparing its signatures with the signatures of known attacks [47]. A State Vector Machine-based security agent detects the new anomalies and stores the information into the database to maintain a log about attacks. CRUZE protects from security attacks: DDoS (HTTP Flood and Zero-Day Attack), Probing (NMAP and Ports sweep), U2R (Buffer Overflow and Rootkits), R2L (IMAP, Guess password and SPY) and DoS (Teardrop, SYN Flood, LAND and Smurf) as discussed in previous research work [47]. Figure 7 (e) shows the comparison of CRUZE, HMRM, CSRE and CSMH in terms of intrusion detection rate with different number of workloads. The value of intrusion detection rate is increasing 
with increase in number of workloads, but CRUZE performs better than HMRM, CSRE and CSMH. The value of intrusion detection rate in CRUZE is $19.20 \%, 21.45 \%$ and $20.86 \%$ more than HMRM, CSRE and CSMH respectively, because CRUZE uses an anomaly detector component i.e. SNORT [47]. It is a signature based system to detect known attacks automatically and stores the signature of attack into database if attack is unknown.

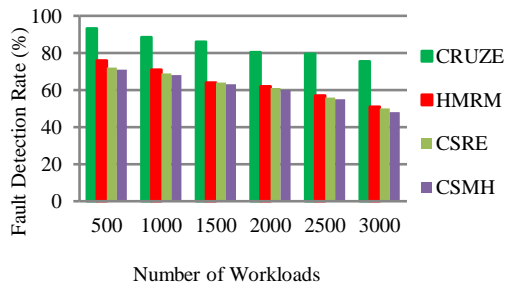

(a)

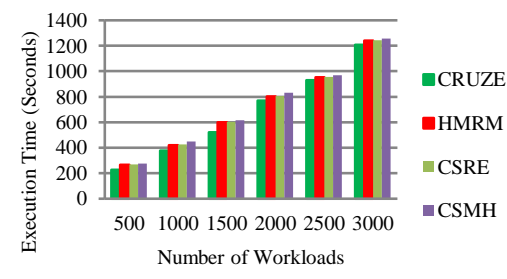

(d)

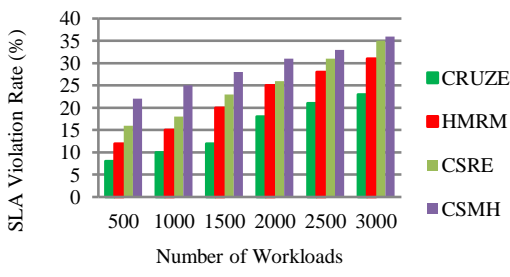

$(\mathrm{g})$

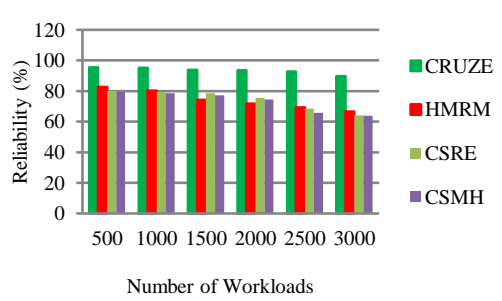

(b)

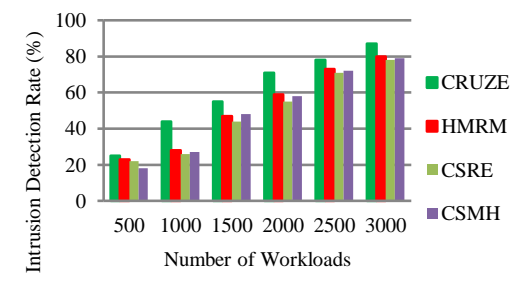

(e)

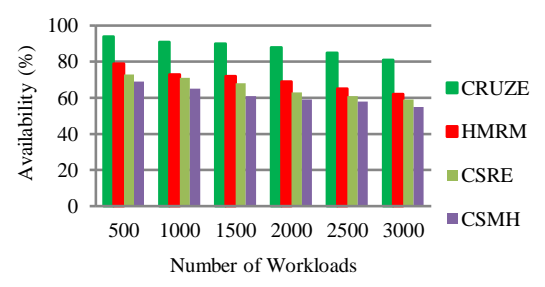

(h)

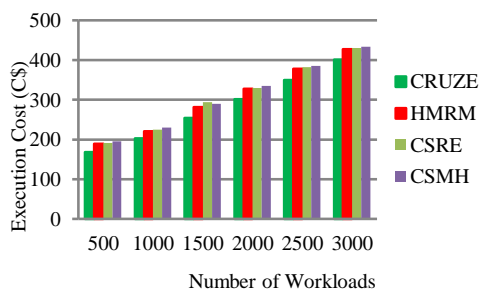

(c)

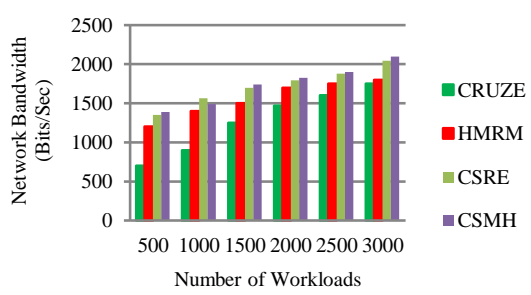

(f)

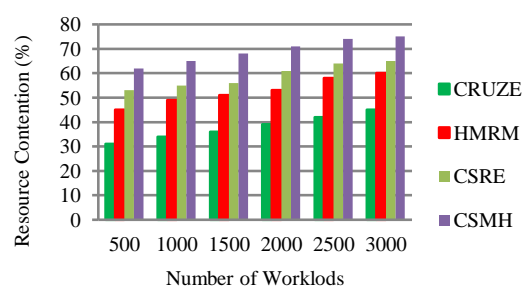

(i)

Figure 7: Comparison of algorithms: (a) Fault Detection Rate, (b) Reliability, (c) Execution Cost, (d) Execution Time, (e) Intrusion Detection Rate, (f) Network Bandwidth, (g) SLA Violation Rate, (h) Availability, (i) Resource Contention. Note: We have considered 36 resources for these results.

We have measured other important QoS parameters such as network bandwidth, SLA violation rate, availability, resource contention to test the performance of CRUZE with different number of workloads and formulas to calculate the value of these QoS parameters is described in previous research work [31] [37] [46]. Figure 7 (f) shows the value of network bandwidth in CRUZE is $14.44 \%, 16.31 \%$ and $18.73 \%$ less than HMRM, CSRE and CSMH respectively. This is because, CRUZE identifies the network faults automatically and it also prevents system from security attacks as discussed above, which improves the network bandwidth of CRUZE as compared to HMRM, CSRE and CSMH. Figure 7 (g) shows the value of SLA violation rate in CRUZE is $23.68 \%, 24.42 \%$ and $27.45 \%$ less than HMRM, CSRE and CSMH respectively. This is because, CRUZE uses admission control and reserve resources for execution of workloads in advance based on their QoS requirements specified in the SLA document. Further, CRUZE outperforms as it regulates the resources at runtime based on the user's new QoS requirements during its execution to avoid SLA violation. Figure 7 (h) shows the value of availability in CRUZE is $12.45 \%, 13.91 \%$ and $15.34 \%$ more than HMRM, CSRE and CSMH respectively. This is expected as the recovering faulty task manages the faults efficiently in CRUZE, which further improves the availability of cloud services. Figure 7 (i) shows the value of resource contention in CRUZE is $17.56 \%, 18.79 \%$ and $19.42 \%$ less than HMRM, CSRE and CSMH respectively. This is expected as the workload execution is done using CRUZE, which is based on QoS parameters based resource provisioning policy (Q-aware). Based on deadline and priority of workload, clustering of workloads is performed, and resources are provisioned for effective scheduling. This is also because of the low variation in execution time across various resources as the resource list that is obtained from the resource provisioning unit is already filtered using Q-aware [31]. 
Case 3 - Capacity Planning: We have considered memory, disk and network utilization as a performance parameter for capacity planning and it is measured in percentage (\%) using [Eq. 14], [Eq. 16] and [Eq. 17] respectively. Figure 8 (a) shows the memory utilization during workload execution for CRUZE, HMRM, CSRE and CSMH and CRUZE executes the same number of workloads with better memory utilization. The value of memory utilization in CRUZE is $24.78 \%, 25.45 \%$ and $25.91 \%$ more than HMRM, CSRE and CSMH respectively. Figure 8 (b) shows the disk utilization during workload execution for CRUZE, HMRM, CSRE and CSMH and CRUZE executes the same number of workloads with better disk utilization. The value of disk utilization in CRUZE is $18 \%, 18.5 \%$ and $19.18 \%$ more than HMRM, CSRE and CSMH respectively. CRUZE gives higher memory and disk utilization as the algorithm consumes resources dynamically based on the requirement of current workloads and unused resources are scaled back to the resource pool. CRUZE keeps only the required number of resources active, thus increasing its utilization efficiency. Figure 8 (c) shows the network utilization during workload execution for CRUZE, HMRM, CSRE and CSMH and CRUZE executes the same number of workloads with better network utilization. The value of network utilization in CRUZE is $12.77 \%, 11.68 \%$ and $12.25 \%$ more than HMRM, CSRE and CSMH respectively because CRUZE performs data transmission with the least packet loss when network utilization reaches at its higher value. CRUZE has FIM-SIM based fault manager (as discussed in Case-1) to detect faults at runtime, which further reduces the occurrence of same network faults in future and it improves network utilization.

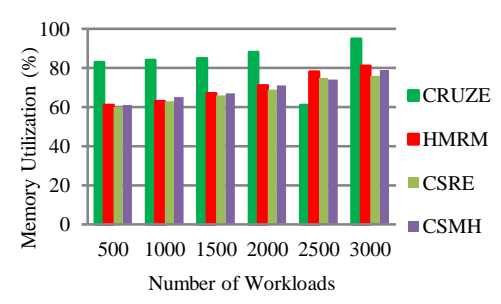

(a)

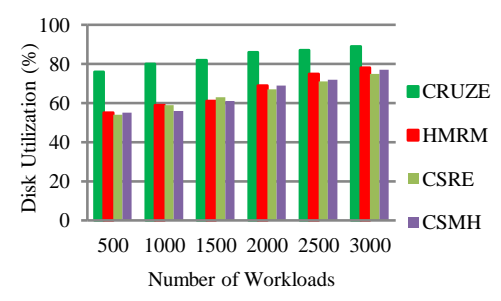

(b)

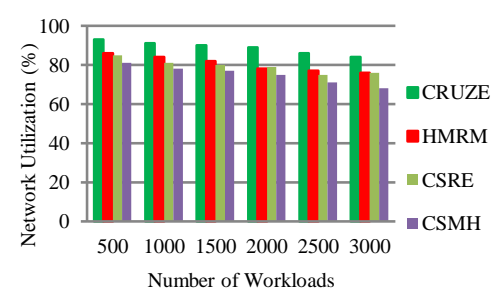

(c)

Figure 8: Comparison of algorithms: (a) Memory Utilization, (b) Disk Utilization, (c) Network Utilization. Note: We have considered 36 resources for these results.

Case 4 - Energy Management: We have evaluated the performance of CRUZE in terms of energy consumption for energy management and used [Eq. 10] to measure the value of energy consumption, which is measured in kilo Watt hour $(\mathrm{kWh})$. Figure 9 (a) shows the variation of energy consumption with different number of workloads and the average value of energy consumption in CRUZE is $17.35 \%, 18.71 \%$ and $20.10 \%$ less than HMRM, CSRE and CSMH respectively. This is because CRUZE executes clustered workloads instead of individual workloads, which minimizes the network traffic and number of switches and further reduces energy consumption.

Case 5 - Virtualization: We have evaluated the performance of CRUZE in terms of CPU utilization and VM Co-Location Cost for virtualization. Figure 9 (b) shows the variation of CPU utilization with different number of workloads for CRUZE, HMRM, CSRE and CSMH. The experimental result show that the average value of CPU utilization in CRUZE is $11.12 \%, 14.45 \%$ and $15.69 \%$ more than HMRM, CSRE and CSMH respectively because best resources are identified using resource provisioning technique for scheduling. Provisioning based scheduling of resources consumes slightly more time initially and then it avoids underutilization and overutilization of resources during scheduling. VM Co-Location Cost is the total cost of VM migration from one cloud datacenter to another [40] [54] and it is calculated using [Eq. 38].

$$
\text { VM CoLocation Cost }=\sum_{i=1}^{n}\left(E_{i}+\mathrm{S}_{i}+\mathrm{A}_{i}+\mathrm{P}_{i}+\mathrm{R}_{i}+\mathrm{C}_{i}\right)
$$

Where $E_{i}$ is Equipment cost (installation cost), $S_{i}$ is Support contract cost (maintenance cost per month), $A_{i}$ is Administrative costs (includes server, storage, network cost per month), $P_{i}$ is Power cost per month (to run CDC), $\mathrm{R}_{i}$ is Rack cost per month, $\mathrm{C}_{i}$ is communication cost and $n$ is the number of VMs. Figure 9 (c) shows the comparison of VM Co-Location Cost for CRUZE, HMRM, CSRE and CSMH to execute different number workloads. The average value of VM Co-Location Cost in CRUZE is 6.25\%, 6.91\% and 7.15\% less than HMRM, CSRE and CSMH respectively because CRUZE identifies the nearest CDC, which consumes more 
renewable energy as compared to other CDCs. The migration of VM to nearest CDC also reduces the communication cost, which further optimize the value of VM Co-Location Cost.

Case 6 - Renewable Energy: We have evaluated the performance of CRUZE in terms of Energy Reuse Effectiveness for renewable energy. Energy Reuse Effectiveness is the ratio of energy (reused) consumed by Cooling, Lighting and IT devices to the total energy consumed by IT devices [35] and described in [Eq. 19]. Figure 9 (d) shows the amount of renewable energy reused during the execution of different number of workloads. The value of energy reuse effectiveness in CRUZE is $17.56 \%, 19.45 \%$ and $20.99 \%$ greater than HMRM, CSRE and CSMH respectively because CRUZE mainly selects the CDC which are utilizing more renewable energy as compared to grid energy. CRUZE manages the energy produced from renewable and nonrenewable sources and sustainable CDCs focuses more on renewable energy sources (solar and wind). To provide reliable services, CDC can prefer grid energy for the execution of deadline-aware workloads.

Case 7 - Thermal-aware Scheduling: We used Computer Room Air Conditioning (CRAC) model based temperature model [34] to test the performance of CRUZE in terms of datacenter temperature for thermalaware scheduling. Datacenter Temperature is the operating temperature of CDC and it is measured in degree Celsius $\left({ }^{\circ} \mathrm{C}\right)$ as described in [Eq. 20]. The variations of the temperature of different hosts (PMs) is measured, monitored and controlled by proactive temperature-aware scheduler. We used an analytical model [64] [65] [66] for the CRAC to measure the temperature of different PMs. Figure 9 (e) shows the comparison of datacenter (CDC) temperature with different number of workloads. The average value of temperature in CRUZE is $13.76 \%, 14.91 \%$ and $15.30 \%$ less than HMRM, CSRE and CSMH respectively. This is because CRUZE optimizes the resource utilization, avoids underloading and overloading of resources and uses minimum energy consumption by reducing the number of components such as number of switches, adapters etc. The other reasons of optimized temperature are effective CRAC-based cooling management [34] and dynamic capacity planning for workload execution. CRUZE automatically switched-off the idle resources in $\mathrm{CDC}$, which also reduces the heat and temperature.

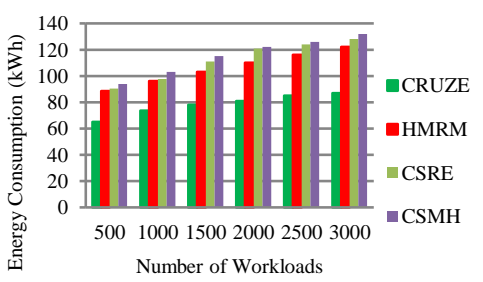

(a)

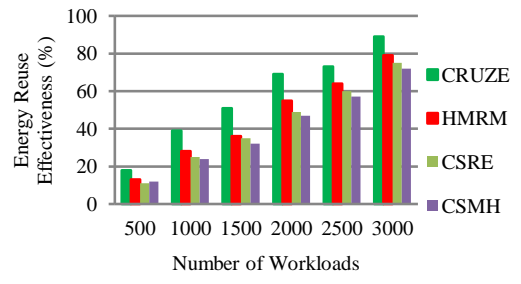

(d)

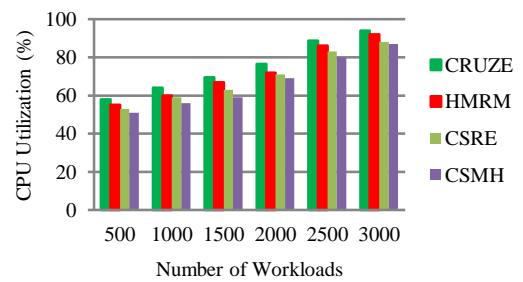

(b)

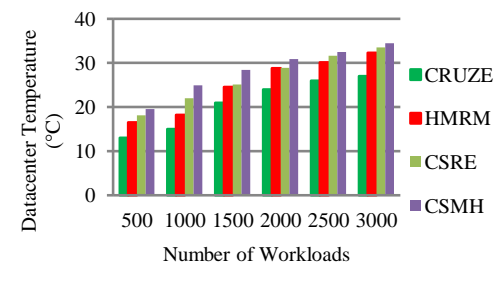

(e)

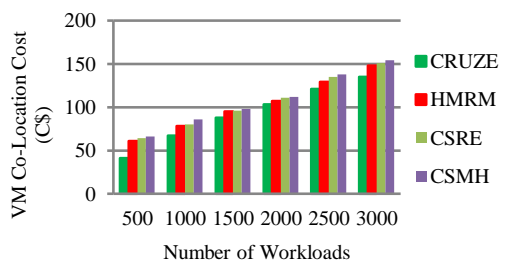

(c)

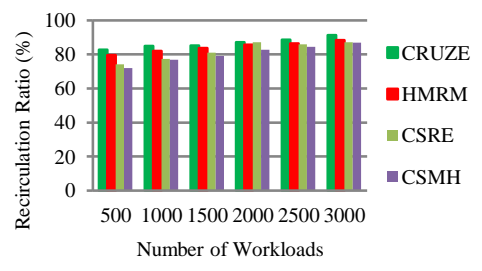

(f)

Figure 9: Performance of different scheduling algorithms: (a) Energy Consumption, (b) CPU Utilization, (c) VM Co-Location Cost, (d) Energy Reuse Effectiveness, (e) Datacenter Temperature, (f) Recirculation Ratio. Note: We have considered 36 resources for these results.

Case 8 - Waste Heat Utilization: We have evaluated the performance of CRUZE in terms of Recirculation Ratio. Recirculation Ratio is the amount of waste-water that flows through the advanced pretreatment component divided by the amount of waste-water that is sent to the final treatment and dispersal component [36] and it is described in [Eq. 20]. Figure 9 (f) shows the value of recirculation ratio for CRUZE, HMRM, CSRE and CSMH during the execution of workloads and the average value of recirculation ratio in CRUZE is $3.42 \% \%, 4.77 \%$ and $4.97 \%$ more than HMRM, CSRE and CSMH respectively. CRUZE performs effective than HMRM, CSRE and CSMH because CRUZE has capability to reuse waste heat in district heating, which further reduces the cost of utilization of waste heat. 
Case 9 - Cooling Management: We have evaluated the performance of CRUZE in terms of Datacenter Cooling System (DCS) Efficiency. DCS Efficiency is the amount of cooling capacity to remove heat per unit of energy it consumes to maintain the cooling of CDC [38] and is described in [Eq. 21]. For cooling management, the district heating management uses water economizer, outside air economizer and chiller plant to control the temperature of CDC. Figure 10 shows the variation of DCS Efficiency with execution of different number of workloads for CRUZE, HMRM, CSRE and CSMH. The average value of DCS Efficiency in CRUZE is $9.98 \%, 10.23 \%$ and $11.56 \%$ more than HMRM, CSRE and CSMH respectively because CRUZE uses district heating management module for effective management of cooling. Figure 11 shows the variation of cooling energy [Eq. 7] with the execution of different number of workloads for CRUZE, HMRM, CSRE and CSMH. The average value of cooling energy in CRUZE is $15.66 \%, 18.31 \%$ and $22.65 \%$ less than HMRM, CSRE and CSMH respectively because CRUZE dynamically switched-on/off the cooling components for different workload intensity, which further reduces the cooling power. Note: We have considered 36 resources for these results.

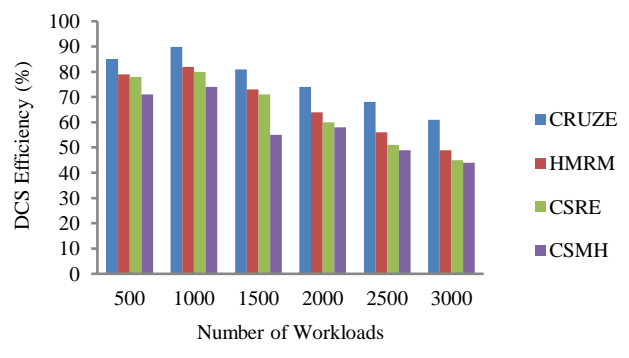

Figure 10: DCS Efficiency vs. Number of Workloads.

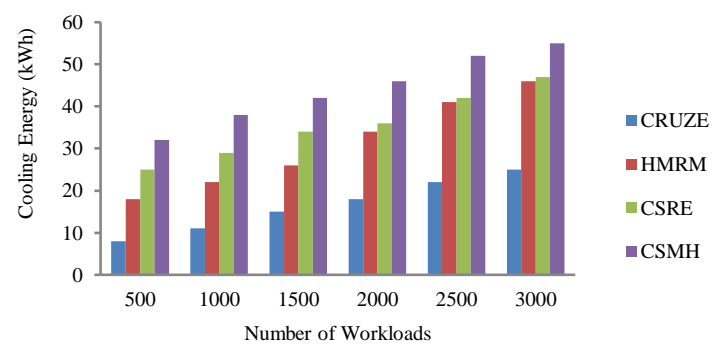

Figure 11: Cooling Energy vs. Number of Workloads.

5.3.1 Comparison of algorithms for different time intervals: We have compared the performance of proposed algorithm with existing algorithms for different time intervals. Figure 12 (a) demonstrates that memory utilization during execution of workloads for CRUZE, HMRM, CSRE and CSMH and the value of memory utilization in CRUZE is $27.77 \%, 28.11 \%$ and $29.12 \%$ more than HMRM, CSRE and CSMH respectively for different time period. Figure 12 (b) shows the variation of energy consumption with different time interval and the average value of energy consumption in CRUZE is $14.46 \%, 15.35 \%$ and $18.86 \%$ less than HMRM, CSRE and CSMH respectively. Figure 12 (c) demonstrates the variation of CPU utilization with different number of workloads for different scheduling techniques. The experimental result show that the average value of CPU utilization in CRUZE is $12.55 \%, 13.91 \%$ and $14.04 \%$ more than HMRM, CSRE and CSMH respectively. This is expected as the workload execution is performed based on QoS-aware resource provisioning policy (Q-aware). Based on deadline of workload, clustering of workloads is performed, and resources are provisioned for effective scheduling. This is also because of the low variation in execution time across various resources as the resource list that is obtained from the resource provisioning unit is already filtered using Q-aware [31]. Based on QoS requirements of a specific workload, resource provisioning consumes little more time to find out the best resources [31], but later it increases the overall performance of CRUZE. Therefore, underutilization and overutilization of CPU will be assuaged or avoided, which reduces the further queuing time. Figure 12 (d) presents the comparison of datacenter (CDC) temperature for different time intervals. The average value of temperature in CRUZE is $8.46 \%, 10.45 \%$ and $13.33 \%$ less than HMRM, CSRE and CSMH respectively. Figure 12 (e) shows the variation of DCS Efficiency for different resource scheduling approaches with different time interval. The average value of DCS Efficiency in CRUZE is $11.46 \%, 12.75 \%$ and $13.01 \%$ more than HMRM, CSRE and CSMH respectively. Figure 12 (f) shows the variation of reliability for different algorithms with different value of time interval. The average value of reliability in CRUZE is $9.21 \%, 9.99 \%$ and $10.21 \%$ more than HMRM, CSRE and CSMH respectively. Figure 12 (g) presents the comparison of execution time for different time intervals. The average value of execution time in CRUZE is $17.65 \%, 18.95 \%$ and $19.63 \%$ less than HMRM, CSRE and CSMH respectively. Figure 12 (h) shows the variation of execution cost for resource management approaches with different time interval. The average value of execution cost in CRUZE is $15.89 \%, 17.72 \%$ and $19.81 \%$ less than HMRM, CSRE and CSMH respectively. Figure 12 (i) shows the variation of SLA violation rate for different resource scheduling algorithms with different time interval. The average value of SLA violation rate in CRUZE is $24.35 \%, 27.29 \%$ and $31.42 \%$ less than HMRM, CSRE and CSMH respectively. Note: We have considered 36 resources and 3000 workloads for these results. 


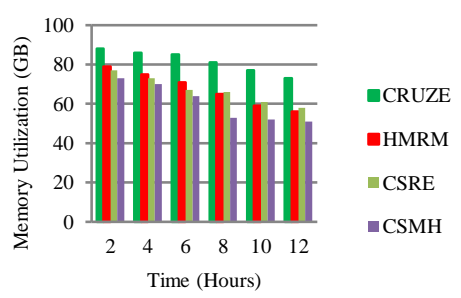

(a)

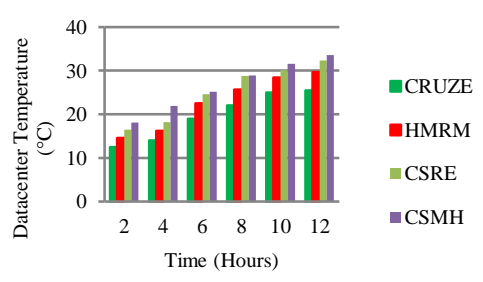

(d)

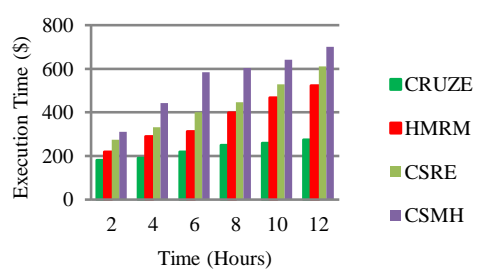

(g)

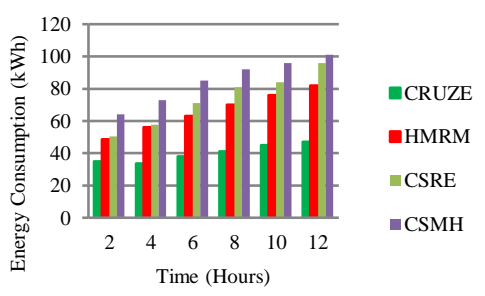

(b)

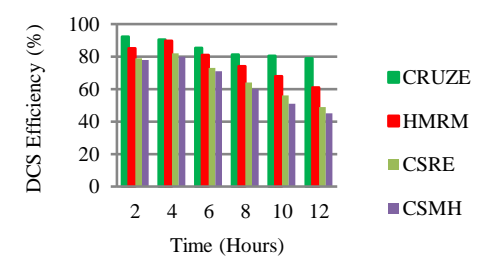

(e)

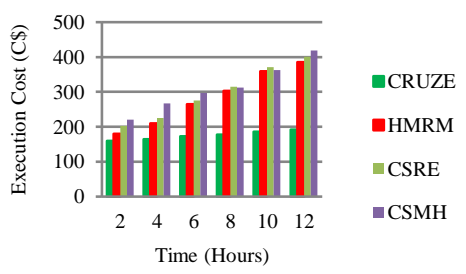

(h)

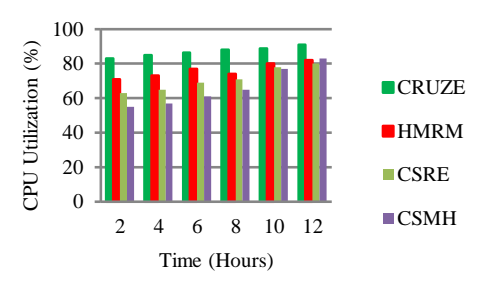

(c)

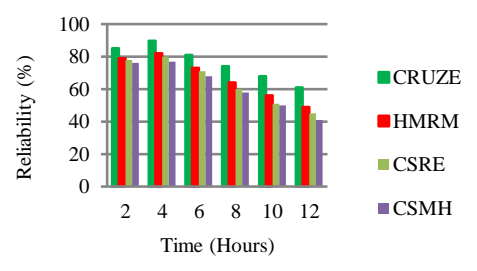

(f)

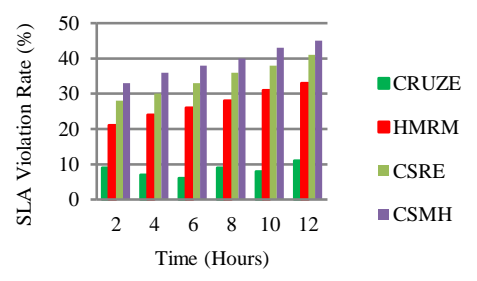

(i)

Figure 12: Comparison of algorithms for different time intervals: (a) Memory Utilization, (b) Energy Consumption, (c) CPU Utilization, (d) Datacenter Temperature, (e) DCS Efficiency, (f) Reliability, (g) Execution Time, (h) Execution Cost, (i) SLA Violation Rate

5.3.2 Trade-off among different Performance Parameters: Figure 13 shows the trade-off among energy consumption, reliability and CPU utilization for execution of workloads using CRUZE. With increasing energy consumption, the value of CPU utilization and reliability is decreasing while reliability of cloud service is increasing with increase in CPU utilization. It is clearly shown that energy consumption is inversely proportional to reliability and CPU utilization, while reliability is proportional to CPU utilization. Note: We have considered 36 resources and 3000 workloads for these results.

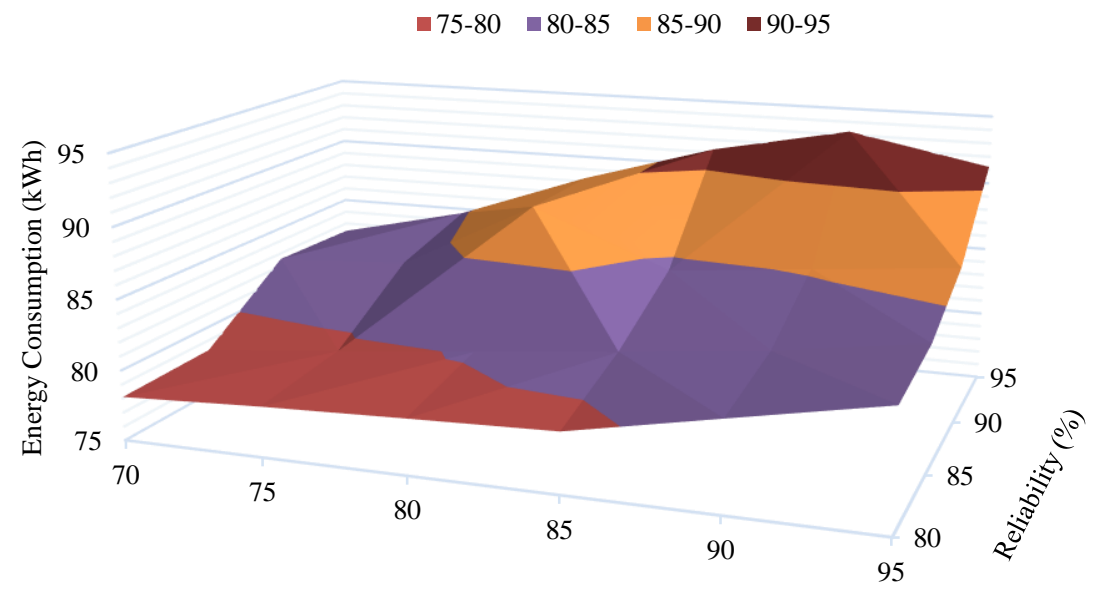

CPU Utilization (\%)

Figure 13: Trade-off among Energy Consumption, Reliability and CPU Utilization 
Figure 14 (a) shows the variation of intrusion detection rate for CRUZE, HMRM, CSRE and CSMH. The value of reliability is increasing as Intrusion detection rate increases for all the approaches, but CRUZE performs better than HMRM, CSRE and CSMH. The average value of Intrusion detection rate in CRUZE is $70 \%$.

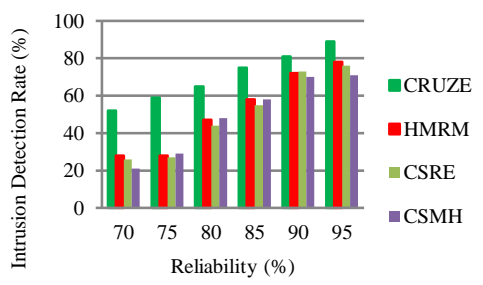

(a)

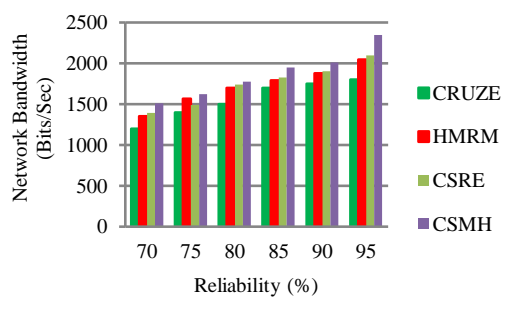

(d)

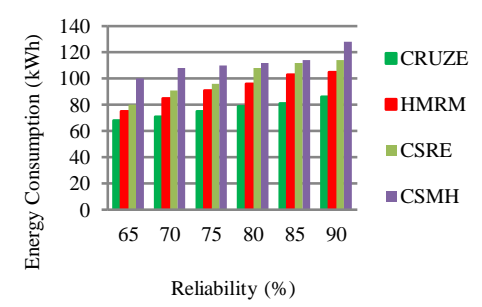

(g)

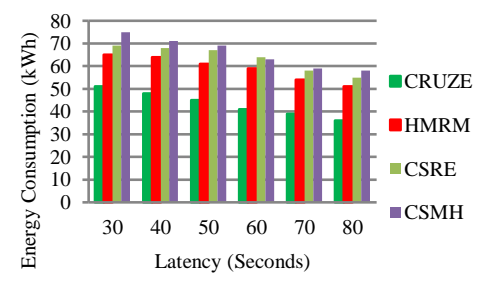

(j)

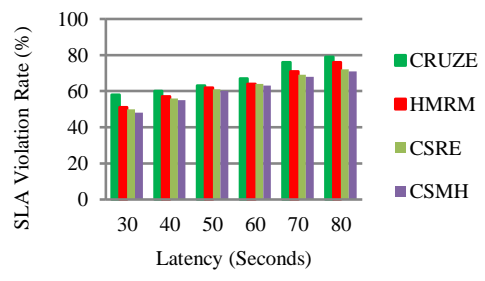

(b)

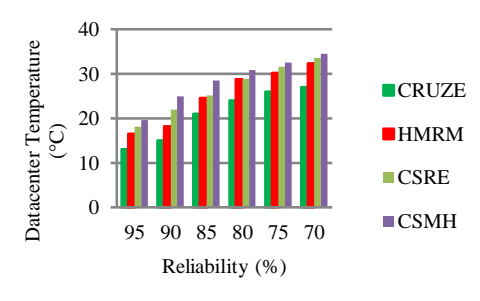

(e)

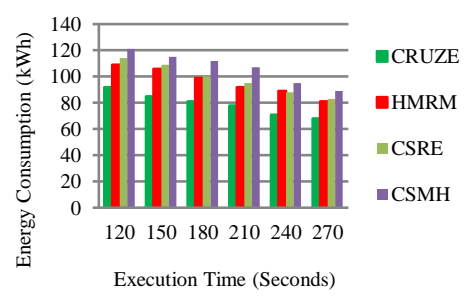

(h)

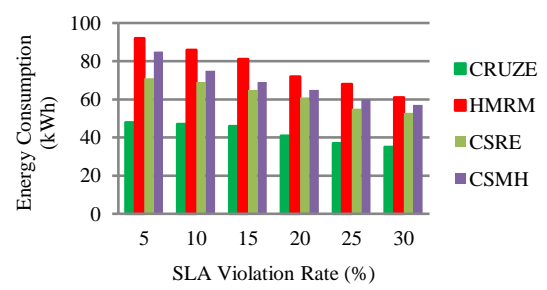

(k)

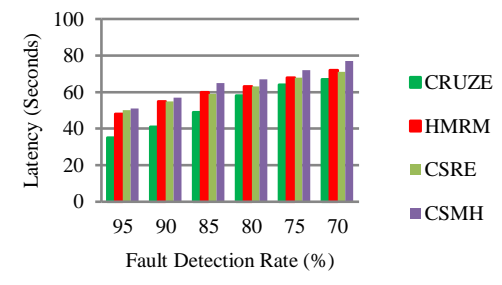

(c)

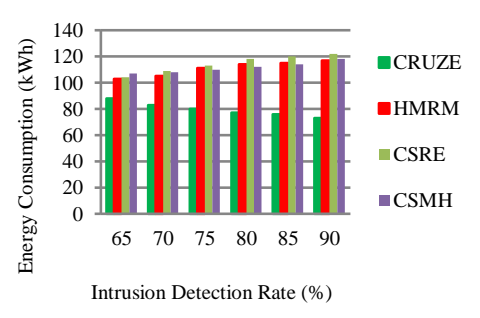

(f)

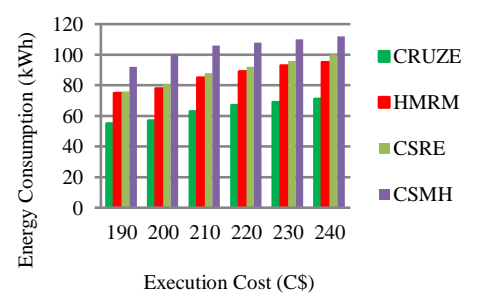

(i)

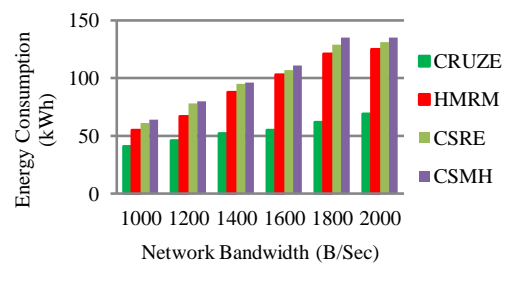

(1)

Figure 14: Trade-off between different Performance Parameters: (a) Intrusion Detection Rate vs. Reliability, (b) SLA Violation Rate vs. Latency, (c) Fault Detection Rate vs. Latency, (d) Network Bandwidth vs. Reliability, (e) Datacenter Temperature vs. Reliability, (f) Energy Consumption vs. Intrusion Detection Rate, (g) Energy Consumption vs. Reliability, (h) Energy Consumption vs. Execu tion Time, (i) Energy Consumption vs. Execution Cost, (j) Energy Consumption vs. Latency, (k) Energy Consumption vs. SLA Violation Rate, (1) Energy Consumption vs. Network Bandwidth

Latency $(L)$ is defined as a difference between expected execution time and actual execution time. We have used following formula to calculate Latency [Eq. 39]:

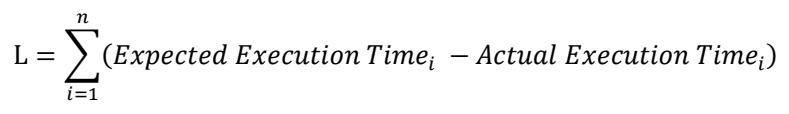

Where $n$ is number of workloads. The value of [Number of workloads $\times$ number on resources] for every workload on resources is calculated from Expectable Time to Compute (ETC) matrix [46]. Columns of ETC matrix demonstrate the estimated execution time for a specific workload while rows on ETC matrix demonstrate the execution time of a workload on every resource. In this research work, the ETC benchmark simulation model is used, which has been 
introduced in [62] to address the problem of resource scheduling. The expected execution time of the workloads can be derived from workload task length or historical trace data [46]. A high variation in execution time of the same workload is generated using the gamma distribution method. In the gamma distribution method, a mean workload execution time and coefficient of variation are used to generate ETC matrix [63]. Table 8 shows a $10 \times 6$ subset of the ETC matrix and results provided in this research work used the matrix of size $90 \times 36$. These are then used to find out the best resource to execute workload with minimum time.

Table 8: A $10 \times 6$ Subset of the ETC Matrix

\begin{tabular}{|c|c|c|c|c|c|c|}
\hline Workloads & $\boldsymbol{r}_{\mathbf{1}}$ & $\boldsymbol{r}_{\mathbf{2}}$ & $\boldsymbol{r}_{\mathbf{3}}$ & $\boldsymbol{r}_{\mathbf{4}}$ & $\boldsymbol{r}_{\mathbf{5}}$ & $\boldsymbol{r}_{\mathbf{6}}$ \\
\hline $\boldsymbol{w}_{\mathbf{1}}$ & 212.14 & 341.44 & 336.65 & 109.66 & 150.46 & 185.58 \\
\hline $\boldsymbol{w}_{\mathbf{2}}$ & 152.61 & 178.26 & 149.78 & 114.26 & 198.92 & 148.69 \\
\hline $\boldsymbol{w}_{\mathbf{3}}$ & 147.23 & 190.23 & 180.26 & 121.65 & 141.65 & 152.69 \\
\hline $\boldsymbol{w}_{\mathbf{4}}$ & 103.62 & 159.63 & 192.85 & 107.69 & 139.89 & 139.36 \\
\hline $\boldsymbol{w}_{\mathbf{5}}$ & 178.65 & 171.35 & 201.05 & 127.65 & 169.36 & 201.66 \\
\hline $\boldsymbol{w}_{\mathbf{6}}$ & 193.62 & 142.65 & 205.36 & 132.26 & 188.33 & 207.72 \\
\hline $\boldsymbol{w}_{\mathbf{7}}$ & 187.24 & 138.23 & 217.58 & 147.69 & 112.39 & 210.98 \\
\hline $\boldsymbol{w}_{\mathbf{8}}$ & 124.13 & 110.65 & 212.39 & 141.26 & 135.88 & 169.35 \\
\hline $\boldsymbol{w}_{\mathbf{9}}$ & 138.56 & 123.65 & 170.26 & 181.65 & 116.61 & 142.87 \\
\hline
\end{tabular}

Figure 14 (b) shows the variation of SLA violation rate for CRUZE, HMRM, CSRE and CSMH with different values of latency. The value of SLA violation rate is increasing as latency increases for all the algorithms, but CRUZE performs better than other algorithms. The average value of SLA violation rate is $67 \%$, which is quite less than HMRM, CSRE and CSMH. Figure 14 (c) shows the variation of latency for CRUZE, HMRM, CSRE and CSMH with different value of fault detection rate. Latency is increasing as the value of fault detection rate decreases for all resource scheduling techniques, but CRUZE performs better than other techniques. The average value of latency in CRUZE is $8.32 \%, 8.49 \%$ and $9.31 \%$ less than HMRM, CSRE and CSMH respectively. The reduction in failure rate, latency and improvement in fault detection rate increases the reliability in CRUZE.

Figure 14 (d) shows the impact of network bandwidth (bits/seconds) on reliability. The value of reliability is increasing as network bandwidth increases for all the approaches, but the average value of network bandwidth in CRUZE is $9.26 \%, 10.55 \%$ and $11.62 \%$ less than HMRM, CSRE and CSMH respectively. Figure 14 (e) shows the impact of datacenter temperature $\left({ }^{\circ} \mathrm{C}\right)$ on reliability. The value of reliability is increasing as datacenter temperature decreases for CRUZE, HMRM, CSRE and CSMH, but CRUZE gives better results as compared to other algorithms. The value of datacenter temperature is $13^{\circ} \mathrm{C}$ in CRUZE at $95 \%$ reliability and the average value of temperature is $21^{\circ} \mathrm{C}$ in CRUZE. Figure 14 (f) shows the variation of energy consumption for different scheduling techniques with different value of intrusion detection rate. The value of energy consumption is increasing as the value of intrusion detection rate decreases for CRUZE, HMRM, CSRE and $\mathrm{CSMH}$, but CRUZE gives better results and the average value of energy consumption in CRUZE is $79.5 \mathrm{kWh}$.

Figure $14(\mathrm{~g})$ shows the trade-off between energy consumption and reliability for all the algorithms and the value of energy consumption is increasing as the value of reliability increases, but CRUZE has better outcome as compared to existing algorithms. The average value of energy consumption in CRUZE is $7.47 \%, 9.42 \%$ and $10.95 \%$ less than HMRM, CSRE and CSMH respectively. Figure 14 (h) shows the impact of execution time on energy consumption for different scheduling algorithms and the value of energy consumption is decreasing as the value of execution time increases for all approaches, but CRUZE consumes less energy as compared to existing techniques. Figure 14 (i) shows the variation of energy consumption for CRUZE, HMRM, CSRE and CSMH with different value of execution cost. The value of execution cost is increasing as the value of energy consumption increases and the average value of energy consumption in CRUZE is $64 \mathrm{kWh}$ approximately. Overall CRUZE performs better than other techniques. The variation of energy consumption with different value of latency is shown in Figure $14(\mathrm{j})$ and it measures the impact of latency on energy consumption and the consumption of energy is increasing as the value of latency decreases for all the resource scheduling approaches, but CRUZE performs better than others. Figure $14(\mathrm{k})$ shows the trade-off between energy consumption and SLA violation rate and the value of energy consumption is increasing as the value of SLA violation rate decreases for all the approaches, but CRUZE performs better than HMRM, CSRE and 
CSMH. The impact of network bandwidth on energy consumption is measured in Figure 14 (1) and the value of energy consumption is increasing as the value of network bandwidth increases. The value of network bandwidth in CRUZE is $16.68 \%, 17.35 \%$ and $17.99 \%$ less than HMRM, CSRE and CSMH respectively.

5.3.3 Straggler Analysis: Due to the increased complexity of modern large-CDCs, certain emerging phenomena, which can directly affect the performance of these systems occur [56]. This is also known as the Long Tail Problem, or the scenario where a small number of task stragglers, negatively affect the time of the workload completion. Task stragglers can occur within any highly parallelized system, which processes workloads consisting of multiple tasks. We have analyzed the performance the effect of various parameters on probability of stragglers. Note: We have considered 36 resources and 3000 workloads for these results. Figure 15 (a) shows the probability of stragglers for different percentage of SLA Violation Rate (SVR). The probability of stragglers is increasing as the value of SVR increases for CRUZE, HMRM, CSRE and CSMH, but CRUZE performs better than other resource scheduling techniques. Figure 15 (b) shows the probability of stragglers for different value of energy consumption. The probability of stragglers is increasing as the value of energy consumption increases for all the algorithms, but the value of straggler probability in CRUZE is $5.45 \%, 5.95 \%$ and $6.36 \%$ less than HMRM, CSRE and CSMH respectively.

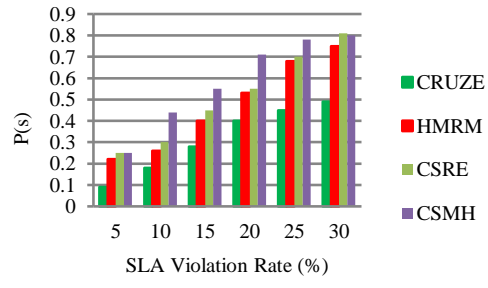

(a)

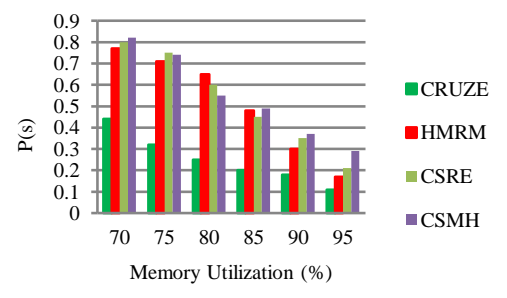

(d)

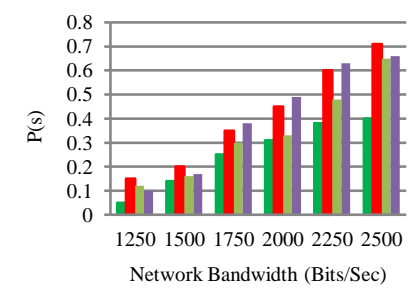

$(\mathrm{g})$

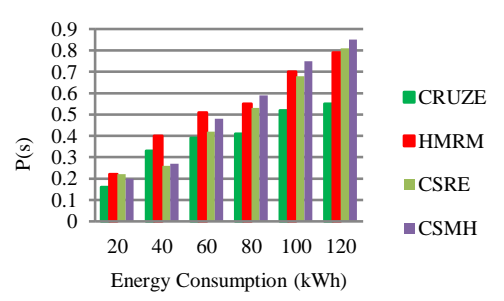

(b)

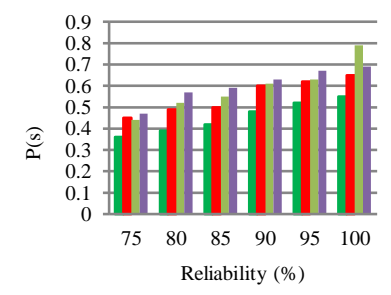

(e)

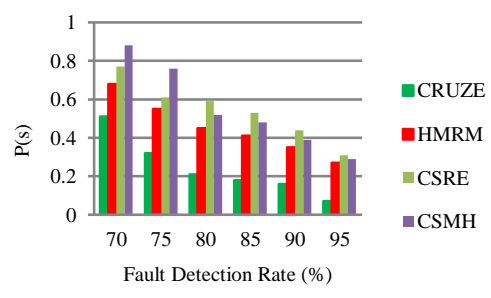

(h)

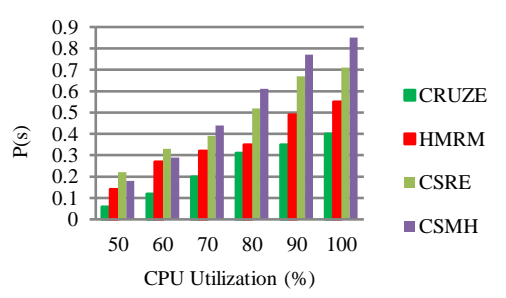

(c)

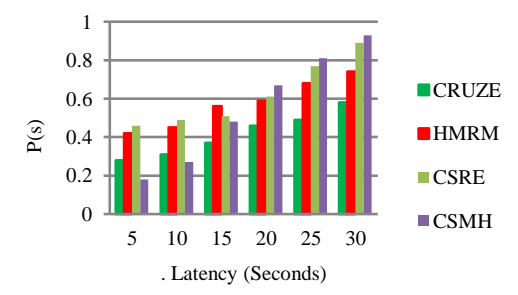

(f)

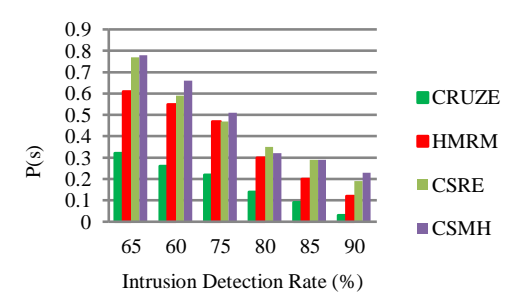

(i)

Figure 15: Analysis of the effect of various performance parameters on Probability of Stragglers (P(s)): a) SLA Violation Rate, b) Energy Consumption, c) CPU Utilization, d) Memory Utilization, e) Reliability, f) Latency, g) Network Bandwidth, h) Fault Detection Rate, and i) Intrusion Detection Rate

Figure 15 (c) shows the probability of stragglers for different percentage of CPU utilization and its average value in CRUZE is 0.24 and it shows the probability of stragglers is increasing as the value of CPU utilization increases for all the resource scheduling techniques, but CRUZE performs better than others. The probability of stragglers is measured for different value of memory utilization as shown in Figure 15 (d) and probability of stragglers is decreasing as the value of memory utilization increases for different scheduling techniques, but CRUZE performs better than other techniques. Figure 15 (e) shows the probability of stragglers for different value of reliability and it is increasing as the value of reliability increases for CRUZE, HMRM, CSRE and CSMH, but CRUZE gives better results than others. 
The probability of stragglers for different value of latency is measured in Figure 15 (f) and it shows the probability of stragglers is increasing as the value of latency increases for CRUZE, HMRM, CSRE and CSMH, but CRUZE performs better than other scheduling techniques. The average value of probability of stragglers in CRUZE is 0.41 . Figure 15 (g) shows the probability of stragglers for different percentage of network bandwidth and CRUZE gives better results than other techniques but the probability of stragglers is increasing as the value of network bandwidth increases for all the scheduling techniques. Figure 15 (h) shows the probability of stragglers for different percentage of fault detection rate. The probability of stragglers is decreasing as the value of fault detection rate increases for CRUZE, HMRM, CSRE and CSMH, but CRUZE performs better than others. Figure 15 (i) shows the probability of stragglers for different percentage of intrusion detection rate. The average value of probability of stragglers in CRUZE is 0.17 and the probability of stragglers is decreasing as the value of intrusion detection rate increases for every approach, but CRUZE performs better than others. The average value of straggler probability in CRUZE is $11.22 \%, 14.01 \%$ and $15.77 \%$ less than HMRM, CSRE and CSMH respectively.

5.3.4 Energy Consumption Analysis: Figure 16 shows the consumption of energy by different components of CDC such as processor, storage, memory, network, cooling and extra using CRUZE as per [Eq. 1]. The processor is most power hungry component of CDC followed by cooling component. The remaining components (storage, memory, network and extra) consumes energy between $2-7 \%$ of total energy consumption by CDC. Note: We have considered 36 resources and 3000 workloads for these results.

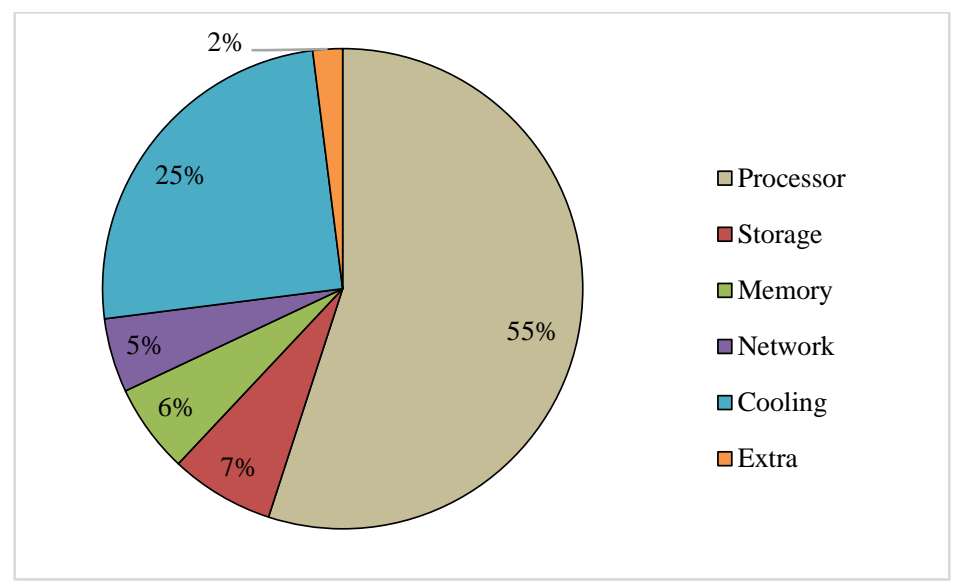

Figure 16: Energy Consumption of different Components of CDC in CRUZE

5.3.5 Convergence of $\mathrm{CO}$ Algorithm: Figure 17 plots the convergence of total energy consumed by $\mathrm{CO}$ algorithm over the number of iterations for different value of Reliability: $95 \%, 90 \%$ and $85 \%$ by executing different number of workloads. Initially the workloads are randomly initialized. Therefore, the total initial energy consumption is very high at $0^{\text {th }}$ iteration. As the algorithm progresses, the convergence is drastic and achieves global minima very quickly. The number of iterations required for the convergence is seen to be $30-45$, for our simulated cloud environment. Note: We have considered 36 resources and 3000 workloads for these results.

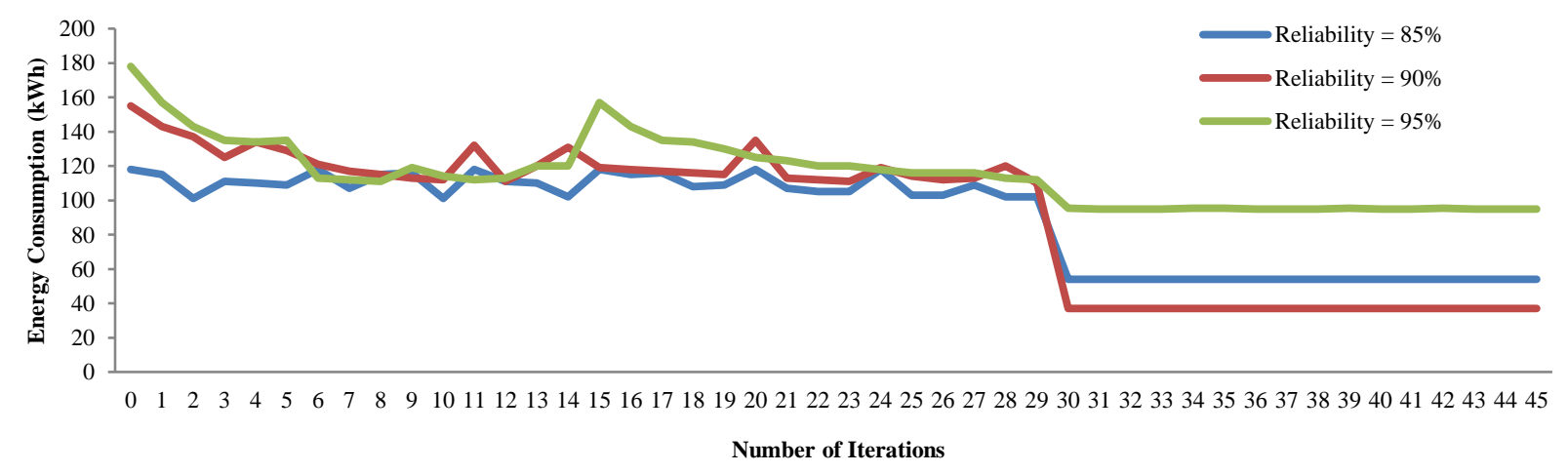

Figure 17: The trend of convergence of $\mathrm{CO}$ with the number of iterations for different value of Reliability 
Table 9 describes summary of experiment statistics and percentage of overall improvement of different performance parameters.

Table 9: Summary of Experimental Statistics and Overall Improvement

\begin{tabular}{|c|c|c|c|c|c|}
\hline \multirow[t]{2}{*}{ Type of Experiment } & \multirow[t]{2}{*}{ Performance Parameter } & \multicolumn{3}{|c|}{ Overall Improvement (\%) } & \multirow{2}{*}{$\begin{array}{c}\text { Average } \\
\text { Improvement (\%) }\end{array}$} \\
\hline & & HMRM & CSRE & CSMH & \\
\hline \multirow{19}{*}{$\begin{array}{l}\text { (Number of } \\
\text { Workloads) }\end{array}$} & Fault Detection Rate & 19.99 & 21.14 & 22.45 & 21.2 \\
\hline & Reliability & 19.07 & 19.75 & 20.98 & 19.9 \\
\hline & Execution Cost & 14.41 & 14.91 & 15.46 & 14.9 \\
\hline & Execution Time & 9.96 & 10.35 & 12.11 & 10.8 \\
\hline & Intrusion Detection Rate & 19.20 & 21.45 & 20.86 & 20.5 \\
\hline & Network Bandwidth & 14.44 & 16.31 & 18.73 & 16.49 \\
\hline & SLA Violation Rate & 23.68 & 24.42 & 27.45 & 25.18 \\
\hline & Availability & 12.45 & 13.91 & 15.34 & 13.9 \\
\hline & Resource Contention & 17.56 & 18.79 & 19.42 & 18.59 \\
\hline & Memory Utilization & 24.78 & 25.45 & 25.91 & 25.38 \\
\hline & Disk Utilization & 18 & 18.5 & 19.18 & 18.56 \\
\hline & Network Utilization & 12.77 & 11.68 & 12.25 & 12.23 \\
\hline & CPU Utilization & 11.12 & 14.45 & 15.69 & 13.75 \\
\hline & Energy Consumption & 17.35 & 18.71 & 20.10 & 18.8 \\
\hline & VM co-Location Cost & 6.25 & 6.91 & 7.15 & 6.8 \\
\hline & Datacenter Temperature & 13.76 & 14.91 & 15.30 & 14.7 \\
\hline & Energy Reuse Effectiveness & 17.46 & 19.45 & 20.99 & 19.3 \\
\hline & Recirculation Ratio & 3.42 & 4.77 & 4.97 & 4.4 \\
\hline & DCS Efficiency & 9.98 & 10.23 & 11.56 & 10.6 \\
\hline \multirow{9}{*}{ (Time in Hours) } & Memory Utilization & 27.77 & 28.11 & 29.12 & 28.3 \\
\hline & Energy Consumption & 14.46 & 15.35 & 18.86 & 16.2 \\
\hline & CPU Utilization & 12.55 & 13.91 & 14.04 & 13.5 \\
\hline & Datacenter Temperature & 8.46 & 10.45 & 13.33 & 10.8 \\
\hline & DCS Efficiency & 11.46 & 12.75 & 13.01 & 12.4 \\
\hline & Reliability & 9.21 & 9.99 & 10.21 & 9.8 \\
\hline & Execution Time & 17.65 & 18.95 & 19.63 & 18.74 \\
\hline & Execution Cost & 15.89 & 17.72 & 19.81 & 17.8 \\
\hline & SLA violation rate & 24.35 & 27.29 & 31.42 & 27.68 \\
\hline
\end{tabular}

5.4.6 Statistical Analysis: Statistical significance of the results has been analyzed by Coefficient of Variation (Coff. of Var.), a statistical method. Coff. of Var. is used to compare to different means and furthermore offer an overall analysis of performance of the framework used for creating the statistics. It states the deviation of the data as a proportion of its average value, and is calculated as follows [Eq. 40]:

$$
\text { Coff. of Var. }=\frac{S D}{\mathrm{M}} \times 100
$$

Where $S D$ is a standard deviation and $M$ is a mean. Coff. of Var. of waiting time of CRUZE, HMRM, CSRE and CSMH is shown in Figure 18 (a). Range of Coff. of Var. $(0.48 \%-1.03 \%)$ for energy consumption approves the stability of CRUZE.
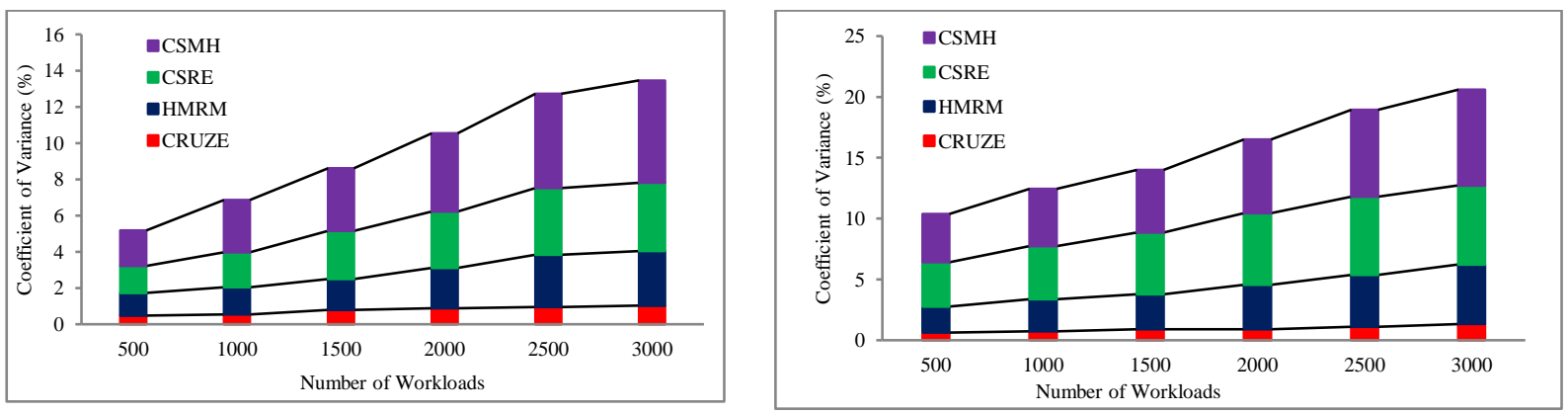

Figure 18: Coefficient of Variation for algorithms (a) energy consumption, (b) reliability 
Coff. of Var. of reliability of CRUZE, HMRM, CSRE and CSMH is shown in Figure 18 (b). Range of Coff. of Var. $(0.63 \%-1.33 \%)$ for reliability approves the stability of CRUZE. Value of Coff. of Var. increases as the number of workloads is increasing. Small value of Coff. of Var. signifies CRUZE is more efficient and stable in resource management in the situations where the number of cloud workloads are changing. CRUZE attained the better results in the cloud for energy consumption and reliability has been studied with respect to number of workloads. This research work is a practical implementation of the conceptual models that we proposed in our previous research work [2] [44].

\section{Summary and Conclusions}

We proposed a Cuckoo Optimization (CO) algorithm based resource scheduling approach called CRUZE, for holistic management of all resources (spanning servers, networks, storage, cooling systems) to improve the energy efficiency and reduce carbon footprints in cloud datacenters and whilst maintaining cloud service reliability by managing the failures (hardware, service, software or resource) dynamically. Furthermore, CRUZE schedules provisioned resources for heterogeneous workload execution and it adjusts the resources at runtime according to the QoS requirements of workloads, which can avoid or assuage under-utilization and over-utilization of resources. Experimental results demonstrate that CRUZE improves the fault detection rate by $15.42 \%$, reliability by $17.11 \%$, intrusion detection rate by $20.46 \%$, CPU utilization by $15.69 \%$, memory utilization by $25.91 \%$, disk utilization by $19.18 \%$, network utilization by $12.25 \%$, energy reuse effectiveness by $20.56 \%$, recirculation ratio by $4.97 \%$ and DCS Efficiency by $11.56 \%$ and it reduces the latency by $8.32 \%$, execution cost by $15.46 \%$, execution time by $12.11 \%$, energy consumption by $20.10 \%$, VM Co-Location Cost by $7.15 \%$ and datacenter temperature by $15.30 \%$ as compared to existing resource management approaches. Finally, the trade-off among energy consumption, reliability and resource utilization for execution of workloads is described.

\subsection{Future Research Directions and Open Challenges}

In the future, we shall explore the applicability of the present model and any potentially needed extensions in the following main directions.

First, the modelling components for workflows analysis and QoS based characterization shall be extended with knowledge of the external context that may inform our holistic management approach. This may require to use additional modeling constructs that help capture the non-functional requirements of each particular application. This is similar to the common technique of prioritization of jobs, for example, depending on the usage context the same workflow can be launched with different priorities.

Second, we shall study possible extensions of the model to include exchange of information and actual hardware, networking, software, storage, heat, and other resources with any other resources from the environment [2]. For example, micro-data centers may be placed in blocks of flats, and the actual heating, ventilation, and air conditioning HVAC (Heating, Ventilation and Air Conditioning) systems [1] may actually use the thermal energy generated by the micro-data center. Moreover, jobs scheduling could happen during periods that inhabitants usually spend at home, which in turn may define the hourly rate for hosting computations [3]. An economy of resources like these may be facilitated by recent development in the area of Blockchain and Smart Contracts, but, it is still necessary to study the theoretical foundations which may potentially lead to energy efficient management of highly distributed Fog computing environments.

Third, many new applications rely on the Internet of Things (IoT) and have particular focus on Big Data management. There is the necessity to implement Big Data pipelines starting from the IoT via Fog and Cloud nodes up to HighPerformance Data Centers [6] [7]. This requires the streaming of significant amounts of data over the network, which in turn represents various management challenges, involving energy efficiency, time-critical operations, and similar [50]. Some of these aspects were tackled by the present study, nevertheless, more in-depth simulations are necessary to study the various arrangements of system components that lead to quasi-optimal states.

Finally, relationship between theory and practice is very important. Benchmarking is an important starting point, which may try to relate the holistic aspects studied in our simulation in real-world practice. For example, various workflow-based applications performing similar calculations, could be related among each other by analyzing the entire hardware and software stack, including virtualization. This may lead to additional improvements of the theoretical basis. 


\section{Acknowledgements}

This research work is supported by the Engineering and Physical Sciences Research Council (EPSRC) - (EP/P031617/1), Melbourne-Chindia Cloud Computing (MC3) Research Network and Australian Research Council (DP160102414). We would like to thank the editor, area editor and anonymous reviewers for their valuable comments and suggestions to help and improve our research paper. We would like to thank Dr. Yogesh Simmhan (IISc Bangalore, India), Dr. Adel Nadjaran Toosi (Monash University, Australia), Mr. Shreshth Tuli (IIT Delhi, India), Mr. Amanpreet Singh (Thapar Institute of Engineering and Technology, India), Mr. Manmeet Singh (Scientist at Indian Institute of Tropical Meteorology, India) and Mr. Damian Borowiec (Lancaster University, UK) for their valuable comments, useful suggestions and discussion to improve the quality of the paper.

\section{References}

[1] Rajkumar Buyya and Sukhpal Singh Gill. "Sustainable Cloud Computing: Foundations and Future Directions." Business Technology \& Digital Transformation Strategies, Cutter Consortium, Vol. 21, no. 6, Pages 1-10, 2018.

[2] Sukhpal Singh Gill and Rajkumar Buyya. "A Taxonomy and Future Directions for Sustainable Cloud Computing: 360 Degree View, ACM Computing Surveys, Vol 51, No. 5, Article No. 104, Pages: 1-33, 2019

[3] Toni Mastelic, Ariel Oleksiak, Holger Claussen, Ivona Brandic, Jean-Marc Pierson, and Athanasios V. Vasilakos. "Cloud computing: Survey on energy efficiency." ACM Computing Surveys, vol. 47, no. 2 (2015): 33.

[4] Junaid Shuja, Abdullah Gani, Shahaboddin Shamshirband, Raja Wasim Ahmad, and Kashif Bilal. "Sustainable cloud datacenters: a survey of enabling techniques and technologies." Renewable and Sustainable Energy Reviews 62 (2016): 195-214.

[5] Yogesh Sharma, Bahman Javadi, Weisheng Si, and Daniel Sun. "Reliability and energy efficiency in cloud computing systems: Survey and taxonomy." Journal of Network and Computer Applications, vol. 74, pp. 66-85, 2016

[6] Li, Xiang, Xiaohong Jiang, Peter Garraghan, and Zhaohui Wu. "Holistic energy and failure aware workload scheduling in Cloud datacenters." Future Generation Computer Systems 78 (2018): 887-900.

[7] Balis, Bartosz, Robert Brzoza-Woch, Marian Bubak, Marek Kasztelnik, Bartosz Kwolek, Piotr Nawrocki, Piotr Nowakowski, Tomasz Szydlo, and Krzysztof Zielinski. "Holistic approach to management of IT infrastructure for environmental monitoring and decision support systems with urgent computing capabilities." Future Generation Computer Systems 79 (2018): 128-143.

[8] Li, Xiang, Peter Garraghan, Xiaohong Jiang, Zhaohui Wu, and Jie Xu. "Holistic virtual machine scheduling in cloud datacenters towards minimizing total energy." IEEE Transactions on Parallel and Distributed Systems (2017).

[9] Natu, Maitreya, Ratan K. Ghosh, Rudrapatna K. Shyamsundar, and Rajiv Ranjan. "Holistic performance monitoring of hybrid clouds: Complexities and future directions." IEEE Cloud Computing 3, no. 1 (2016): 72-81.

[10] Pérez, Juan F., Lydia Y. Chen, Massimo Villari, and Rajiv Ranjan. "Holistic Workload Scaling: A New Approach to Compute Acceleration in the Cloud." IEEE Cloud Computing 5, no. 1 (2018): 20-30.

[11] Luo, Changqing, Laurence T. Yang, Pan Li, Xia Xie, and Han-Chieh Chao. "A holistic energy optimization framework for cloud-assisted mobile computing." IEEE Wireless Communications 22, no. 3 (2015): 118-123.

[12] Liu, Bingwei, Yu Chen, Erik Blasch, Khanh Pham, Dan Shen, and Genshe Chen. "A holistic cloud-enabled robotics system for real-time video tracking application." In Future Information Technology, pp. 455-468. Springer, Berlin, Heidelberg, 2014.

[13] Guzek, Mateusz, Dzmitry Kliazovich, and Pascal Bouvry. "A holistic model for resource representation in virtualized cloud computing data centers." In Cloud Computing Technology and Science (CloudCom), 2013 IEEE 5th International Conference on, vol. 1, pp. 590-598. IEEE, 2013.

[14] Ferrer, Ana Juan, Francisco HernáNdez, Johan Tordsson, Erik Elmroth, Ahmed Ali-Eldin, Csilla Zsigri, RaüL Sirvent et al. "OPTIMIS: A holistic approach to cloud service provisioning." Future Generation Computer Systems 28, no. 1 (2012): 66-77.

[15] Feller, Eugen, Cyril Rohr, David Margery, and Christine Morin. "Energy management in IaaS clouds: a holistic approach." In Cloud Computing (CLOUD), 2012 IEEE 5th International Conference on, pp. 204-212. IEEE, 2012.

[16] Ao Zhou, Shangguang Wang, Zibin Zheng, Ching-Hsien Hsu, Michael R. Lyu and Fangchun Yang. "On cloud service reliability enhancement with optimal resource usage." IEEE Transactions on Cloud Computing 4, no. 4, 452-466, 2016.

[17] Mingqiang Li, Chuan Qin, Jingwei Li and Patrick PC Lee. "CDStore: Toward reliable, secure, and cost-efficient cloud storage via convergent dispersal." IEEE Internet Computing 20, no. 3, 45-53, 2016.

[18] Fatemeh Azimzadeh and Fatemeh Biabani. "Multi-objective job scheduling algorithm in cloud computing based on reliability and time." In Web Research (ICWR), 2017 3th International Conference on, pp. 96-101. IEEE, 2017.

[19] Deepak Poola, Kotagiri Ramamohanarao, and Rajkumar Buyya, Enhancing Reliability of Workflow Execution Using Task Replication and Spot Instances, ACM Transactions on Autonomous and Adaptive Systems (TAAS), Volume 10, Number 4, Article No. 30, Pages: 1-21, ISSN:1556-4665, ACM Press, New York, USA, February 2016.

[20] Chenhao Qu, Rodrigo N. Calheiros and Rajkumar Buyya, A Reliable and Cost-Efficient Auto-Scaling System for Web Applications Using Heterogeneous Spot Instances, Journal of Network and Computer Applications (JNCA), Volume 65, Pages: 167-180, ISSN: 1084-8045, Elsevier, Amsterdam, The Netherlands, April 2016.

[21] Xunyun Liu, Aaron Harwood, Shanika Karunasekera, Benjamin Rubinstein and Rajkumar Buyya, E-Storm: Replication-based State Management in Distributed Stream Processing Systems, Proceedings of the 46th International Conference on Parallel Processing (ICPP 2017, IEEE CS Press, USA), Bristol, UK, August 14-17, 2017.

[22] Latiff, Muhammad Shafie Abd, Syed Hamid Hussain Madni, and Mohammed Abdullahi. "Fault tolerance aware scheduling technique for cloud computing environment using dynamic clustering algorithm." Neural Computing and Applications 29, no. 1 (2018): 279-293.

[23] S. Shahdi-Pashaki, Ehsan Teymourian, Vahid Kayvanfar, GH M. Komaki and A. Sajadi. "Group technology-based model and cuckoo optimization algorithm for resource allocation in cloud computing." IFAC-PapersOnLine 48, no. 3 (2015): 1140-1145.

[24] R. Sundarrajan, and V. Vasudevan. "An Optimization Algorithm for Task Scheduling in Cloud Computing Based on Multi-Purpose Cuckoo Seek Algorithm." In International Conference on Theoretical Computer Science and Discrete Mathematics, pp. 415-424. Springer, Cham, 2016.

[25] Mohammad Javad Abbasi and Mehrdad Mohri "Scheduling Tasks in the Cloud Computing Environment with the Effect of Cuckoo Optimization Algorithm", SSRG International Journal of Computer Science and Engineering (SSRG - IJCSE), V3 (8), 1-9 August 2016 
[26] Nima Jafari Navimipour and Farnaz Sharifi Milani. "Task scheduling in the cloud computing based on the cuckoo search algorithm." International Journal of Modeling and Optimization 5, no. 1 (2015): 44.

[27] Syed Hamid Hussain Madni, Muhammad Shafie Abd Latiff and Shafi Muhammad Abdulhamid. "Optimal Resource Scheduling for IaaS Cloud Computing using Cuckoo Search Algorithm." Sains Humanika 9, no. 1-3 (2017).

[28] Ramin Rajabioun, "Cuckoo optimization algorithm", Applied Soft Computing 11(8) (2011): 5508-5518.

[29] Xin-She Yang. "Swarm intelligence based algorithms: a critical analysis." Evolutionary intelligence 7, no. 1 (2014): 17-28.

[30] Rodrigo N. Calheiros, Rajiv Ranjan, Anton Beloglazov, César AF De Rose and Rajkumar Buyya. "CloudSim: a toolkit for modeling and simulation of cloud computing environments and evaluation of resource provisioning algorithms." Software: Practice and experience 41, no. 1 (2011): 23-50.

[31] Singh, Sukhpal, and Inderveer Chana. "Q-aware: Quality of service based cloud resource provisioning." Computers \& Electrical Engineering 47 (2015): 138-160.

[32] Gill, Sukhpal Singh, Inderveer Chana, and Rajkumar Buyya. "IoT Based Agriculture as a Cloud and Big Data Service: The Beginning of Digital India." Journal of Organizational and End User Computing (JOEUC) 29, no. 4 (2017): 1-23.

[33] Kouki, Yousri, and Thomas Ledoux. "Sla-driven capacity planning for cloud applications." In Cloud Computing Technology and Science (CloudCom), 2012 IEEE 4th International Conference on, pp. 135-140. IEEE, 2012.

[34] Moore, Justin D., Jeffrey S. Chase, Parthasarathy Ranganathan, and Ratnesh K. Sharma. "Making Scheduling" Cool": Temperature-Aware Workload Placement in Data Centers." In USENIX annual technical conference, General Track, pp. 61-75. 2005.

[35] Tschudi, B. I. L. L., O. T. T. O. Vangeet, J. Cooley, and D. Azevedo. "ERE: A metric for measuring the benefit of reuse energy from a data center." White Paper 29 (2010).

[36] Karellas, Sotirios, and Konstantinos Braimakis. "Energy-exergy analysis and economic investigation of a cogeneration and trigeneration ORC-VCC hybrid system utilizing biomass fuel and solar power." Energy conversion and management 107 (2016): 103-113.

[37] Gill, Sukhpal Singh, Inderveer Chana, Maninder Singh, and Rajkumar Buyya. "CHOPPER: an intelligent QoS-aware autonomic resource management approach for cloud computing." Cluster Computing (2017): 1-39.

[38] Liu, Zhenhua, Yuan Chen, Cullen Bash, Adam Wierman, Daniel Gmach, Zhikui Wang, Manish Marwah, and Chris Hyser. "Renewable and cooling aware workload management for sustainable data centers." In ACM SIGMETRICS Performance Evaluation Review, vol. 40, no. 1, pp. 175-186. ACM, 2012.

[39] Gill, Sukhpal Singh, and Rajkumar Buyya. "Resource Provisioning Based Scheduling Framework for Execution of Heterogeneous and Clustered Workloads in Clouds: from Fundamental to Autonomic Offering." Journal of Grid Computing (2018): 1-33.

[40] Oxley, Mark A., Eric Jonardi, Sudeep Pasricha, Anthony A. Maciejewski, Howard Jay Siegel, Patrick J. Burns, and Gregory A. Koenig. "Rate-based thermal, power, and co-location aware resource management for heterogeneous data centers." Journal of Parallel and Distributed Computing 112 (2018): 126-139.

[41] L.A. Barroso, J.Clidaras, U. Hoelze, The Datacenter as a Computer: An Introduction to the Design of Warehouse-Scale Machines, Synthesis Lectures on Computer Architecture, July 2013.

[42] P.Garraghan. I. Solis Moreno, P. Townend, J. Xu, An Analysis of Failure-related Energy Waste in a Large-scale Cloud Environment, IEEE Transactions on Emerging Topics in Computing, pp. 166-180, 2014.

[43] Li, Xiang, Xiaohong Jiang, and Yanzhang He. "Virtual Machine Scheduling Considering Both Computing and Cooling Energy." In High Performance Computing and Communications, 2014 IEEE 6th Intl Symp on Cyberspace Safety and Security, 2014 IEEE 11th Intl Conf on Embedded Software and Syst (HPCC, CSS, ICESS), 2014 IEEE Intl Conf on, pp. 244-247. IEEE, 2014.

[44] Sukhpal Singh Gill and Rajkumar Buyya, "Failure Management for Reliable Cloud Computing: A Taxonomy, Model and Future Directions", Computing in Science and Engineering, IEEE, 2018. DOI: 10.1109/MCSE.2018.2873866

[45] Nita, Mihaela-Catalina, Florin Pop, Mariana Mocanu, and Valentin Cristea. "FIM-SIM: Fault injection module for CloudSim based on statistical distributions."Journal of telecommunications and information technology 4 (2014): 14.

[46] Sukhpal Singh Gill, Inderveer Chana, Maninder Singh and Rajkumar Buyya, RADAR: Self-Configuring and Self-Healing in Resource Management for Enhancing Quality of Cloud Services, Concurrency and Computation: Practice and Experience (CCPE), Volume 31, No. 1, Pages: 1-29, 2019

[47] Gill, Sukhpal Singh, and Rajkumar Buyya. "SECURE: Self-Protection Approach in Cloud Resource Management." IEEE Cloud Computing 5, no. 1 (2018): 60-72.

[48] Singh, Sukhpal, and Inderveer Chana. "QRSF: QoS-aware resource scheduling framework in cloud computing." The Journal of Supercomputing 71, no. 1 (2015): 241-292.

[49] Moreno, Ismael Solis, Peter Garraghan, Paul Townend, and Jie Xu. "Analysis, modeling and simulation of workload patterns in a large-scale utility cloud." IEEE Transactions on Cloud Computing 2, no. 2 (2014): 208-221.

[50] Kaur, Amanpreet, V. P. Singh, and Sukhpal Singh Gill. "The Future of Cloud Computing: Opportunities, Challenges and Research Trends." In 2nd International Conference on I-SMAC (IoT in Social, Mobile, Analytics and Cloud), pp. 213-219. IEEE, 2018.

[51] Deb, Kalyanmoy. "Constrained Multi-objective Evolutionary Algorithm." In Evolutionary and Swarm Intelligence Algorithms, pp. 85-118. Springer, Cham, 2019.

[52] Guitart, Jordi. "Toward sustainable data centers: a comprehensive energy management strategy." Computing 99, no. 6 (2017): $597-615$.

[53] Singh, Sukhpal, and Inderveer Chana. "A survey on resource scheduling in cloud computing: Issues and challenges." Journal of grid computing 14, no. 2 (2016): 217-264.

[54] Youn, Chan-Hyun, Min Chen, and Patrizio Dazzi. Cloud Broker and Cloudlet for Workflow Scheduling. Springer Singapore, 2017.

[55] Gill, Sukhpal Singh, Peter Garraghan, and Rajkumar Buyya. "ROUTER: Fog Enabled Cloud based Intelligent Resource Management Approach for Smart Home IoT Devices."" Journal of Parallel and Distributed Computing 154 (2019): 125-138.

[56] Garraghan, Peter, Xue Ouyang, Renyu Yang, David McKee, and Jie Xu. "Straggler root-cause and impact analysis for massive-scale virtualized cloud datacenters." IEEE Transactions on Services Computing (2016).

[57] Chang, Che-Wei, Chun-Yi Liu, and Chuan-Yue Yang. "Energy-efficient heterogeneous resource management for wireless monitoring systems." Journal of Systems and Software 131 (2017): 168-180.

[58] Taherizadeh, Salman, Andrew C. Jones, Ian Taylor, Zhiming Zhao, and Vlado Stankovski. "Monitoring self-adaptive applications within edge computing frameworks: A state-of-the-art review." Journal of Systems and Software 136 (2018): 19-38.

[59] Singh, Sukhpal, and Inderveer Chana. "Consistency verification and quality assurance (CVQA) traceability framework for SaaS." In 2013 3rd IEEE International Advance Computing Conference (IACC), pp. 1-6. IEEE, 2013.. 
[60] D. Sitaram, H. L. Phalachandra, G. S, S. H V and S. TP, "Energy efficient data center management under availability constraints," 2015 Annual IEEE Systems Conference (SysCon) Proceedings, Vancouver, BC, 2015, pp. 377-381.

[61] L. Luo, H. Li, X. Qiu and Y. Tang, "A Resource Optimization Algorithm of Cloud Data Center Based on Correlated Model of Reliability, Performance and Energy," 2016 IEEE International Conference on Software Quality, Reliability and Security Companion (QRS-C), Vienna, 2016, pp. 416-417.

[62] Tracy D. Braun, Howard Jay Siegel, Noah Beck, Ladislau L. Bölöni, Muthucumaru Maheswaran, Albert I. Reuther, James P. Robertson et al. "A comparison of eleven static heuristics for mapping a class of independent tasks onto heterogeneous distributed computing systems." Journal of Parallel and Distributed computing 61, no. 6 (2001): 810-837.

[63] Shoukat Ali, Howard Jay Siegel, Muthucumaru Maheswaran, Debra Hensgen and Sahra Ali. "Representing task and machine heterogeneities for heterogeneous computing systems." Tamkang J Sci Eng, 3, no. 3 (2000): 195-207.

[64] Zhang S, Chatha K S. Approximation Algorithm for the Temperature Aware Scheduling Problem. Proceedings of International Conference on Computer-Aided Design. 2007:281-288.

[65] Tang Qinghui, Gupta Sandeep Kumar S, Varsamopoulos Georgios. Energy-efficient thermal-aware task scheduling for homogeneous highperformance computing data centers: A cyber-physical approach. IEEE Transactions on Parallel and Distributed Systems. 2008;19(11):1458-1472

[66] Lazic, Nevena, Craig Boutilier, Tyler Lu, Eehern Wong, Binz Roy, M. K. Ryu, and Greg Imwalle. "Data center cooling using model-predictive control." In Advances in Neural Information Processing Systems, pp. 3814-3823. 2018.

[67] Singh, Sukhpal, and Inderveer Chana. "EARTH: Energy-aware autonomic resource scheduling in cloud computing." Journal of Intelligent \& Fuzzy Systems 30, no. 3 (2016): 1581-1600.

[68] Möbius, Christoph, Waltenegus Dargie, and Alexander Schill. "Power consumption estimation models for processors, virtual machines, and servers." IEEE Transactions on Parallel and Distributed Systems 25, no. 6 (2014): 1600-1614.

[69] Grozev, Nikolay, and Rajkumar Buyya. "Performance modelling and simulation of three-tier applications in cloud and multi-cloud environments." The Computer Journal 58, no. 1 (2013): 1-22.

[70] Lebre, Adrien, Arnaud Legrand, Frédéric Suter, and Pierre Veyre. "Adding storage simulation capacities to the simgrid toolkit: Concepts, models, and api." In 2015 15th IEEE/ACM International Symposium on Cluster, Cloud and Grid Computing, pp. 251-260. IEEE, 2015.

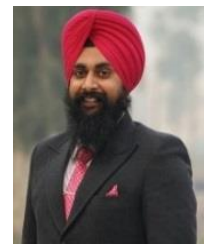

\section{Author's Biography}

Dr. Sukhpal Singh Gill is currently working as a Research Associate at School of Computing and Communications, Lancaster University, UK. Dr. Gill was a Postdoctoral Research Fellow at Cloud Computing and Distributed Systems (CLOUDS) Laboratory, School of Computing and Information Systems, The University of Melbourne, Australia. He was a recipient of several awards, including the Distinguished Reviewer Award from Software: Practice and Experience (Wiley), 2018, and served as the PC member for venues such as UCC, SE-CLOUD, ICCCN, ICDICT and SCES. His one review paper has been nominated and selected for the ACM 21 st annual Best of Computing Notable Books and Articles as one of the notable items published in computing - 2016. He has published more than 45 papers as a first author. Dr. Gill also worked in Computer Science and Engineering Department of Thapar Institute of Engineering and Technology (TIET), Patiala, India, as a Lecturer. He obtained the Degree of Master of Engineering in Software Engineering (Gold Medalist), as well as a Doctoral Degree specialization in Autonomic Cloud Computing from TIET. He was a DST (Department of Science \& Technology) Inspire Fellow during Doctorate and worked as a SRF-Professional on DST Project, Government of India. His research interests include Cloud Computing, Software Engineering, Internet of Things, Big Data and Fog Computing. For further information on Dr. Gill, please visit: www.ssgill.in

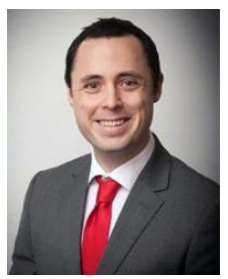

Dr. Peter Garraghan is a Lecturer (Assistant Professor) in the School of Computing \& Communications, Lancaster University. He received his PhD in Computer Science from the University of Leeds, UK. He has industrial experience building large-scale systems and his research interests include distributed systems, Cloud datacenters, dependability, and energy-efficient computing, where he has published over $35+$ refereed papers.

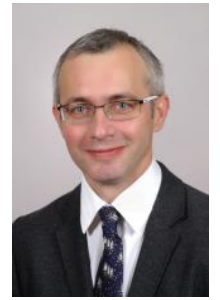

Dr. Vlado Stankovski a Professor (at College) in Computer and Information Science and Informatics in Commerce at University of Ljubljana. Vlado Stankovski was awarded his Eng.Comp.Sc., M.Sc. and Ph.D. degrees in computer science from the University of Ljubljana in 1995, 2000 and 2009, respectively. He began his career in 1995 as consultant and later as project manager with the FujitsuICL Corporation in Prague. From 1998-2002 he worked as researcher at the University Medical Centre in Ljubljana. From 2003 on, he is with the Department of Construction Informatics at the University of Ljubljana. He lectures in undergraduate computer science subjects. Vlado Stankovski's research interests are in semantic and distributed-computing technologis. He has been the technical manager of the FP6 DataMiningGrid project and financial manager of the FP6 InteliGrid project. He also participates in Slovene national grid-related projects, such as: GridForum.si, AgentGrid and SiGNet. His past experience is in applications of machine learning techniques to engineering and medical problems. 


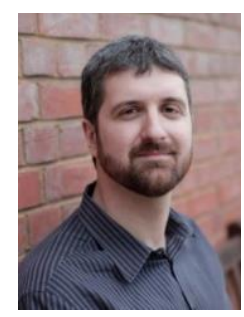

Dr. Giuliano Casale received the Ph.D. degree in computer engineering from Politecnico di Milano, Italy, in 2006. He joined the Department of Computing, Imperial College London, U.K., in 2010, where he is currently a Senior Lecturer in modeling and simulation. He was a Scientist with SAP Research, U.K. and as a Consultant in the capacity planning industry. He teaches and does research in performance engineering, cloud computing, and Big data, topics on which he has published over 120 refereed papers. He has served on the technical program committee of over 80 conferences and workshops and as the co-chair for conferences in the area of performance engineering such as ACM SIGMETRICS/Performance. He is a member of the IFIP WG 7.3 group on Computer Performance Analysis and since 2015 he has been serving in the ACM SIGMETRICS Board of Directors. He was a recipient of several awards, including the Best Paper Award at ACM SIGMETRICS 2017, and served as the Program Chair for venues such as ACM SIGMETRICS/Performance, MASCOTS, ICAC, ICPE, and QEST.

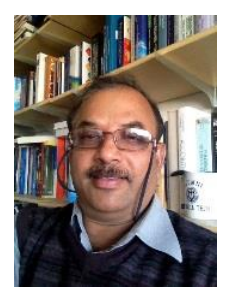

Dr. Ruppa K. Thulasiram (Tulsi) (M'00-SM'09) received the Ph.D. degree from the Indian Institute of Science, Bangalore, India. He is a Professor and the Director of the Computational Finance Laboratory, Department of Computer Science, University of Manitoba, Winnipeg, MB, Canada. He spent years with Concordia University, Montreal, QC, Canada; Georgia Institute of Technology, Atlanta, GA, USA; and the University of Delaware, Newark, DE, USA, as a Postdoctoral Researcher, Research Staff, and Research Faculty before taking up a position with the University of Manitoba. He has graduated many students with M.Sc. and Ph.D. degrees. He has developed a curriculum for the cross-disciplinary computational finance course at the University of Manitoba for both graduate and senior undergraduate levels. He has authored or co-authored many papers in the areas of high-temperature physics, gas dynamics, combustion, computational finance, and grid/cloud computing. His current research interest includes grid/cloud computing, computational finance, cloud resources management, computational intelligence, ad hoc networks, and scientific computing. His research has been funded by the Natural Sciences and Engineering Research Council (NSERC) of Canada.Dr. Thulasiram has been an Associate Editor for the IEEE Transactions on Cloud Computing, and International Journal of Aerospace Innovations (MultiSceince Publishing). He is a member of the Editorial Board of many journals including the International Journal of Computational Science and Engineering. He has been a guest editor of many journals such as Parallel Computing, Concurrency and Computation Practice and Experience, the International Journal of Parallel, Embedded and Distributed Systems, and the Journal of Supercomputing for special issues. He was the recipient of many Best Paper Awards.

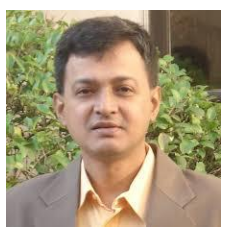

Dr. Soumya K. Ghosh is a Professor in the Department of Computer Science and Engineering, Indian Institute of Technology, Kharagpur (IIT Kharagpur), India. Soumya K. Ghosh (M’05) received the M. Tech. and Ph.D. degrees from the Department of Computer Science and Engineering, IIT Kharagpur, Kharagpur, India, in 1996 and 2002, respectively. He was with the Indian Space Research Organization, Bengaluru, India. He has authored or coauthored more than 200 research papers in reputed journals and conference proceedings. His current research interests include spatial data science, spatial web services, and cloud computing.

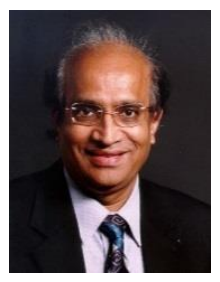

Dr. Ramamohanarao (Rao) Kotagiri received his $\mathrm{PhD}$ from Monash University. He was awarded the Alexander von Humboldt Fellowship in 1983. He has been at the University Melbourne since 1980 and was appointed as a professor in computer science in 1989. Rao held several senior positions including Head of Computer Science and Software Engineering, Head of the School of Electrical Engineering and Computer Science at the University of Melbourne and Research Director for the Cooperative Research Center for Intelligent Decision Systems. He served or serving on the Editorial Boards of the Computer Journal, Universal Computer Science, IEEE TKDE, VLDB Journal and International Journal on Data Privacy. Rao is a Fellow of the Institute of Engineers Australia, a Fellow of Australian Academy Technological Sciences and Engineering and a Fellow of Australian Academy of Science.

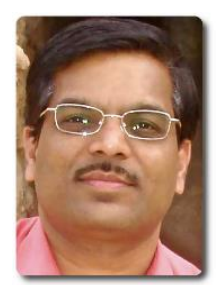

Dr. Rajkumar Buyya is a Redmond Barry Distinguished Professor and Director of the Cloud Computing and Distributed Systems (CLOUDS) Laboratory at the University of Melbourne, Australia. He is also serving as the founding CEO of Manjrasoft, a spin-off company of the University, commercializing its innovations in Cloud Computing. He served as a Future Fellow of the Australian Research Council during 2012-2016. He has authored over 625 publications and seven text books including "Mastering Cloud Computing" published by McGraw Hill, China Machine Press, and Morgan Kaufmann for Indian, Chinese and international markets respectively. He also edited several books including "Cloud Computing: Principles and Paradigms" (Wiley Press, USA, Feb 2011). He is one of the highly cited authors in computer science and software engineering worldwide (h-index=124+, g-index=281, 80000+ citations). Microsoft Academic Search Index ranked Dr. Buyya as \#1 author in the world (2005-2016) for both field rating and citations evaluations in the area of Distributed and Parallel Computing. "A Scientometric Analysis of Cloud Computing Literature" by German scientists ranked Dr. Buyya as the World's Top-Cited (\#1) Author and the World's Most-Productive (\#1) Author in Cloud Computing. Recently, Dr. Buyya is recognized as a "Web of Science Highly Cited Researcher" in both 2016 and 2017 by Thomson Reuters, a Fellow of IEEE, and Scopus Researcher of the Year 2017 with Excellence in Innovative Research Award by Elsevier for his outstanding contributions to Cloud computing. He served as the founding Editor-in-Chief of the IEEE Transactions on Cloud Computing. He is currently serving as Editorin-Chief of Journal of Software: Practice and Experience, which was established over 45 years ago. For further information on Dr. Buyya, please visit his cyberhome: www.buyya.com 